\title{
Can equations of equilibrium predict all physical equilibria? A case study from Field Dislocation Mechanics
}

\author{
Amit Das ${ }^{\text {a }}$, Amit Acharya ${ }^{a^{*}}$, Johannes Zimmer ${ }^{b}$, Karsten Matthies ${ }^{b}$ \\ ${ }^{a}$ Civil and Environmental Engineering, Carnegie Mellon University, Pittsburgh, \\ PA 15213, USA \\ ${ }^{b}$ Department of Mathematical Sciences, University of Bath, Bath BA27AY, United \\ Kingdom
}

*Email: acharyaamit@.cmu.edu Phone: 412-268-4566

\section{Abstract}

Numerical solutions of a one dimensional model of screw dislocation walls (twist boundaries) are explored. The model is an exact reduction of the 3D system of partial differential equations of Field Dislocation Mechanics. It shares features of both Ginzburg-Landau (GL) type gradient flow equations as well as hyperbolic conservation laws, but is qualitatively different from both. We demonstrate such similarities and differences in an effort to understand the equation through simulation. A primary result is the existence of spatially non-periodic, extremely slowly evolving (quasi-equilibrium) cell-wall dislocation microstructures practically indistinguishable from equilibria, which however cannot be solutions to the equilibrium equations of the model, a feature shared with certain types of GL equations. However, we show that the class of quasi-equilibria comprising spatially non-periodic microstructure consisting of fronts is larger than that of the GL equations associated with the energy of the model. In addition, under applied strain-controlled loading, a single dislocation wall is shown to be capable of moving as a localized entity as expected in a physical model of dislocation dynamics, in contrast to the associated GL equations. The collective evolution of the quasi-equilibrium cell-wall microstructure exhibits a yielding-type behavior as bulk plasticity ensues, and the effective stress-strain response under loading is found to be rate-dependent. The numerical scheme employed is non-conventional since wave-type behavior has to be accounted for, and interesting features of two different schemes are discussed. Interestingly, a stable scheme conjectured by us to produce a non-physical result in the present context nevertheless suggests a modified continuum model that appears to incorporate apparent intermittency.

Key words: Dislocation mechanics, plasticity, microstructure, quasi-equilibria 


\section{Introduction}

This paper studies a simple model of Field Dislocation mechanics to test its potential for the study of individual and collective dislocation dynamics in the presence of nonconvex crystal elasticity. The simplified mathematical model used in our study is formulated in Acharya (2010) using the equations of Field Dislocation Mechanics and traveling wave solutions and equilibria of this model have been studied analytically in Acharya, Matthies and Zimmer (AMZ) (2010). Here we concentrate on general time-dependent approximations of solutions to this model not restricted to traveling waves and carry out our investigation numerically. A rigorous analysis of global existence and uniqueness of a more general class of equations to which the present equation belongs is provided in Acharya and Tartar (2011).

Our results include evolutions that illustrate the coexistence of both positive and negative dislocations in a quasi-equilibrium state under no load. A variety of patterned equilibria are observed. Stick-slip behavior in average stress-strain response is observed in the presence of loading. The stress-strain response under loading displays strain-rate hardening.

An upwinding and a Friedrichs scheme are used to solve the equation. The influence of these numerical schemes on the behavior of equilibrium profiles under no load and on the motion of dislocations is also analyzed.

\section{Model}

\subsection{Physical description}

Consider an infinite cylinder of rectangular cross-section as shown in Fig. 1. The cylinder is subjected to simple shear by applying spatially uniform displacement boundary condition on the top surface and by holding the bottom surface fixed. Homogeneous loading in the model is prescribed by specifying $g$ which denotes the applied shear strain. $u$ is the displacement on the top surface of the cylinder in 
the $y$ direction. It is assumed that at any time $t$ all fields vary only along the axis of the cylinder. The only non-zero component of stress field is $\tau$ which represents the shear stress in the $y$ direction on planes with normal in the $z$ direction. Likewise, the only non-zero component of plastic distortion is $\varphi$ which represents plastic shearing in the $y$ direction on planes with normal in the $z$ direction. The spatial gradient of plastic distortion $\varphi_{x}$ represents the only component of the dislocation density field representing screw dislocations with line and Burgers vector along the $y$ direction. The temporal gradient of plastic distortion $\varphi$ is denoted by $\varphi_{t}$.

In Acharya (2010) a time dependent 3D system of equations governing the rate of plastic distortion tensor is formulated. The above assumptions are utilized to derive an exact $1 \mathrm{D}$ reduction of those equations resulting in the following governing equation as described in Acharya (2010), and AMZ (2010):

$$
\varphi_{t}=\frac{\left|\varphi_{x}\right|}{B}\left[\varepsilon \varphi_{x x}+\tau(g-\varphi)\right]
$$

where $B$ (dislocation 'drag') and $\varepsilon$ (core energy 'modulus') are material parameters, discussed further in Sec. 1.4.

Consider a periodic-cubic function $\hat{\tau}$ of the following form

$$
\hat{\tau}(a)=-\frac{\mu}{2\left(\frac{\bar{\varphi}}{2}\right)^{2}} a\left[a^{2}-\left(\frac{\bar{\varphi}}{2}\right)^{2}\right]
$$

extended periodically beyond $\left[-\frac{\bar{\varphi}}{2}, \frac{\bar{\varphi}}{2}\right], \mu>0$.

The function $\tau$ appearing in the PDE (1) is defined in terms of $\hat{\tau}$ as

$$
\tau(g-\varphi)=-\hat{\tau}\left(g-\varphi-\frac{\bar{\varphi}}{2}\right)
$$

It satisfies 


$$
\tau(0)=0 \quad ; \quad \mu=\tau^{\prime}(0)>0,
$$

which implies that the shear stress is zero and shear modulus is positive at zero elastic strain.

Fig. 2 (a) and (b) show the $\hat{\tau}$ and $\tau$ function respectively. Fig. 2 (c) is a plot of energy corresponding to the stress function $\hat{\tau}$. The wells are at $x=0$ and $x= \pm \bar{\varphi}$ where the energy is minimum.

\subsection{Notation}

The following notation is used in the numerical scheme described below. Consider the discrete field variable $\varphi^{k}\left(x_{h}\right)$. The superscript $k$ indicates the time increment. The distance from the origin of the domain is indicated by the symbol $x_{h}$ inside the brackets () , which is the position of the $h^{\text {th }}$ node. Discrete spatial and temporal derivatives are denoted by subscripts. The discrete spatial derivative of $\varphi$ at time increment $k$ and location $x_{h}$ is denoted by $\varphi_{x}^{k}\left(x_{h}\right)$. Likewise, the discrete temporal derivative of $\varphi$ at time increment $k$ and location $x_{h}$ is denoted by $\varphi_{t}^{k}\left(x_{h}\right)$. The discrete double spatial derivative is denoted by $\varphi_{x x}^{k}\left(x_{h}\right)$. The

notation $\tau^{k}\left(g-\varphi^{k}\left(x_{h}\right)\right)$ denotes the value of the function $\tau$ for time increment $k$ at spatial location.

\subsection{Numerical Scheme}

We follow Acharya et al. (2004) in formulating the scheme utilized to deal with wave like response. The linearized form of (1) is obtained formally by taking the first variation of (1): 


$$
\begin{aligned}
\delta \varphi_{t}^{k}\left(x_{h}\right)= & -\left(\frac{-\operatorname{sgn}\left(\varphi_{x}^{k}\left(x_{h}\right)\right)}{B}\right)\left[\varepsilon \varphi_{x x}^{k}\left(x_{h}\right)+\tau^{k}\left(g-\varphi^{k}\left(x_{h}\right)\right)\right] \delta \varphi_{x}^{k}\left(x_{h}\right) \\
& +\frac{\left|\varphi_{x}^{k}\left(x_{h}\right)\right|}{B}\left[\varepsilon \delta \varphi_{x x}^{k}\left(x_{h}\right)\right] \\
& +\frac{\left|\varphi_{x}^{k}\left(x_{h}\right)\right|}{B}\left[-\tau^{\prime k}\left(g-\varphi^{k}\left(x_{h}\right)\right)\right] \delta \varphi^{k}\left(x_{h}\right),
\end{aligned}
$$

The first term of (5) provides linearized wave-like behavior. A corresponding velocity $c^{k}\left(x_{h}\right)$ is obtained as follows

$$
\begin{gathered}
\begin{aligned}
& \delta \varphi_{t}^{k}\left(x_{h}\right)=-c^{k}\left(x_{h}\right) \delta \varphi_{x}^{k}\left(x_{h}\right)+\frac{\left|\varphi_{x}^{k}\left(x_{h}\right)\right|}{B}\left[\varepsilon \delta \varphi_{x x}^{k}\left(x_{h}\right)\right] \\
&+\frac{\left|\varphi_{x}^{k}\left(x_{h}\right)\right|}{B}\left[-\tau^{\prime k}\left(g-\varphi^{k}\left(x_{h}\right)\right)\right] \delta \varphi^{k}\left(x_{h}\right), \\
& \text { where } c^{k}\left(x_{h}\right)=\left(\frac{-\operatorname{sgn}\left(\varphi_{x}^{k}\left(x_{h}\right)\right)}{B}\right)\left[\varepsilon \varphi_{x x}^{k}\left(x_{h}\right)+\tau^{k}\left(g-\varphi^{k}\left(x_{h}\right)\right)\right] .
\end{aligned}
\end{gathered}
$$

To calculate $c^{k}\left(x_{h}\right)$ for a time increment $k$ at a spatial location $x_{h}$ the required values of $\varphi_{x}^{k}\left(x_{h}\right)$ and $\varphi_{x x}^{k}\left(x_{h}\right)$ are computed using central finite differences

$$
\begin{aligned}
& \frac{\varphi^{k}\left(x_{h+1}\right)-\varphi^{k}\left(x_{h-1}\right)}{2 d} \\
& \frac{\varphi^{k}\left(x_{h+1}\right)-2 \varphi^{k}\left(x_{h}\right)+\varphi^{k}\left(x_{h-1}\right)}{d^{2}} .
\end{aligned}
$$

Once $c^{k}\left(x_{h}\right)$ is obtained, depending on the sign of $c^{k}\left(x_{h}\right)$ the discrete value of $\varphi_{x}^{k}\left(x_{h}\right)$ is updated as follows:

$$
\begin{aligned}
\varphi_{x}^{k} & :=\frac{\varphi^{k}\left(x_{h+1}\right)-\varphi^{k}\left(x_{h}\right)}{d} & \text { if } & c^{k}\left(x_{h}\right)<0 \\
\varphi_{x}^{k} & :=\frac{\varphi^{k}\left(x_{h}\right)-\varphi^{k}\left(x_{h-1}\right)}{d} & \text { if } & c^{k}\left(x_{h}\right)>0 \\
\varphi_{x}^{k} & :=\frac{\varphi^{k}\left(x_{h+1}\right)-\varphi^{k}\left(x_{h-1}\right)}{2 d} & \text { if } & c^{k}\left(x_{h}\right)=0 .
\end{aligned}
$$


where $d$ is the element size of the domain. The values of $\varphi$ used to compute $\varphi_{x}$ are picked from the upwind side of wave motion and hence this scheme is known as 'upwind scheme'.

For the time-step determination, each of the three terms on RHS of (5) is considered. The minimum of the three time steps corresponding to the three mechanisms is used to compute the time step increment $\Delta t^{k}$ as shown below.

$$
\Delta t^{k}=\min _{h}\left(\frac{d}{c^{k}\left(x_{h}\right)}, \frac{d^{2} B}{\varepsilon\left|\varphi_{x}^{k}\left(x_{h}\right)\right|}, \frac{B}{\left|\varphi_{x}^{k}\left(x_{h}\right)\right|\left[-\tau^{\prime k}\left(g-\varphi^{k}\left(x_{h}\right)\right)\right]}\right) .
$$

$\varphi$ is updated according to the following scheme.

$$
\frac{\varphi^{k+1}\left(x_{h}\right)-\varphi^{k}\left(x_{h}\right)}{\Delta t^{k}}=\frac{\left|\varphi_{x}^{k}\left(x_{h}\right)\right|}{B}\left[\begin{array}{l}
\tau\left(g^{k}-\varphi^{k}\left(x_{h}\right)\right)+ \\
\varepsilon \frac{\varphi^{k}\left(x_{h+1}\right)-2 \varphi^{k}\left(x_{h}\right)+\varphi^{k}\left(x_{h-1}\right)}{d^{2}}
\end{array}\right]
$$

\subsection{Problem Setup}

The total length $L=1000 b$ where $b=4.05 \times 10^{-4} \mu m$ is the magnitude of the Burgers vector. Other parameters are shear modulus $\mu=23 G P a, \varepsilon=\frac{\mu b^{2}}{4}$ and $\bar{\varphi}=0.05$. Neumann boundary conditions $\varphi_{x}=0$ are applied at the ends of the domain (mostly for simplicity, since most of our conclusions relate to patterns in the interior of the body; we note that while a vanishing flux of plastic strain is physically natural boundary condition, a vanishing dislocation density is not so). Time is expressed in units of $\frac{b B}{\mu}$ and the velocity units are $\frac{\mu}{B}$.

\section{Characterization of Equilibrium Profiles}




\subsection{Equilibrium profiles connecting wells}

A variety of possible equilibrium solutions for the governing equation of plastic flow are analyzed in this section. All homogeneous $\varphi$ profiles are equilibria. The following non-homogeneous profiles connecting energy wells $\varphi=0$ and $\varphi=-\bar{\varphi}$ are equilibria in an infinite domain (Acharya, 2010, section 4.1).

$$
\varphi_{e q}=g-\frac{\bar{\varphi}}{2} \pm \frac{\bar{\varphi}}{2} \tanh \left(\sqrt{\frac{\mu}{4 \varepsilon} x}\right)
$$

We utilize this solution to check whether it suffices to generate an approximate equilibrium solution on a finite domain. Fig. 3 illustrates the verification of a spatially inhomogenous equilibrium profile. The analytical solution (11) profile with the center at $x=500 b$ shown below is prescribed as an initial condition. The applied strain $g$ is set to zero to simulate no applied loads. There is no noticeable change in the $\varphi$ profile during the simulation.

Fig. 4 (a) shows the plot of $\varphi_{x}$ for the analytical solution profile. It represents a single wall of dislocations. Fig. 4 (b) is a plot of the stress corresponding to the dislocation wall. High stresses are observed in the dislocation core. The stress decreases rapidly to zero away from the core.

\subsection{Equilibrium profiles between wells}

The motivation for the questions posed here comes from the analytical results of AMZ (2010) in which all possible equilibria of the model are characterized.by a phase portrait analysis. The equilibrium profiles discussed in the previous section are profiles generated by (11) which connect the energy wells i.e. the transition layer goes from one energy minimum at $\varphi=0$ to another energy minimum at $\varphi=-\bar{\varphi}$. In addition to those profiles, profiles of the type shown in Fig. 5 are analyzed. When the lower and upper levels of the transition layer are equidistant from the energy wells there is no change in the form of $\varphi$ profile when simulation 
is performed with $g$ set to zero. When the lower and upper levels of the transition layer are not equidistant from the energy wells then the resulting profile is not equilibrium. The sequence of evolution of this profile is shown in Fig. 6. The profile moves with time towards the right and eventually exits the domain. We note that all of these solutions discussed in this section are continuous in space.

\subsection{Multiple transition layers and quasi-equilibria}

The figures (7 and 8) show a section of the domain, the same pattern being repeated throughout the entire domain and set by the initial conditions. Irrespective of whether two dislocations of the same or opposite sign are adjacent to each other, the corresponding $\varphi$ profile is a quasi-equilibrium when the ends of the profile connect the wells.

In comparison to the above case, a different trend is observed for the case when the ends of transition layers are between wells. When a positive and a negative dislocation are adjacent to each other and the ends of the transition layer of the $\varphi$ profile are between wells then the form of the $\varphi$ profile remains unchanged as shown in Fig. 9. However, when two dislocations of same sign are adjacent to each other and the ends of the transition layer of the $\varphi$ profile are between wells then the profile shown in Fig. 10 is found not to be in equilibrium.

As discussed in AMZ (2010) multiple, full Burgers vector walls, i.e. transition layers connecting adjacent wells of the energy, separating dislocation-free cells are physically desirable features as idealized representations of observed cell-wall microstructures or pile-ups. While in our idealized setting only screw-walls are considered, the walls in fatigue cell-wall microstructures consist of edge dislocation dipoles e.g. Ahmed, Wilkinson, Roberts (1997), i.e. they consist of alternate positive and negative walls. In pile-ups, they all have the same sign. Interestingly, as explained in AMZ (2010) multiple transition layers connecting wells are not possible as a strict equilibrium in the model. This is due to the reason that an equilibrium transition layer connecting two wells is represented by a heteroclinic orbit in the phase plane $\varphi_{x}$ vs. $\varphi-g$ (Fig. 3, AMZ) joining two 
equilibria and therefore the connection is possible only in infinite 'time' which in this context translates to an infinite spatial domain. However, the results obtained using the full dynamic equations (Figs. 7 and 8) show such profiles involving multiple transition layers connecting wells that appear to be in equilibrium for all practical purposes. These results represent the remarkable fact that such transition layer patterns evolve extremely slowly but cannot be predicted from the corresponding equilibrium equations. In this paper, we term such profiles as quasi-equilibria. This behavior of our equations is analogous to the rigorously analyzed behavior of Ginzburg Landau equations in Carr and Pego (1989). The results observed in Figs. 9 and 10 are in full agreement with the analysis in AMZ (2010).

\section{Dislocation Motion}

In the cases studied so far static equilibrium solutions were analyzed with $g$, the applied strain, set to zero. In this section dislocation motion is studied by subjecting the profile to constant homogenous load by specifying a non-zero $g$. The importance of the appropriate solution scheme necessary to model dislocation motion is emphasized and the trend of the resulting dislocation velocity profiles is discussed.

The profile corresponding to (11) with $g$ set to zero is prescribed as an initial condition. Dislocation motion for this simulation is shown in Fig. 11 (a). The profile (dislocation) moves towards the left or right depending upon the sign of $g$. The profile does not exhibit traveling wave characteristics since its form is distorted during motion.

It is important to note that the upwind scheme explained in (8) is essential for predicting dislocation motion. Without the upwind criteria, the profile remains stationary even under non-zero loads. 
Fig. 11 (b) is a plot of average dislocation velocity $v^{*}$ versus applied strain. The profile corresponding to (11) with $g$ set to zero is prescribed as an initial condition. For each constant positive $g$, a simulation is performed for a total time of 10 units. This time is chosen to ensure the transition layer profile does not exit the domain before the completion of the simulation. The average velocity is calculated for each $g$ by measuring the total distance traversed by the transition layer profile and dividing it by the total time. The average velocity versus applied strain curve starts with what appears to be a zero slope for small strains and then varies approximately as square of the applied strain at larger strains.

\section{Multiple Dislocation Walls}

In order to study the behavior of multiple dislocation walls, the initial condition imposed is as shown in Fig. 12. A repeated sequence of a collection of sine waves separated by a wide spacing in between is specified over the entire domain. Each collection consists of three sine waves, each having a wavelength of $25 b$. The spacing between each collection of sine wave is equal to $75 \mathrm{~b}$. A simulation is performed for the no loading case with applied strain, $g$, set to zero. The amplitude of the sine waves is equal to $\bar{\varphi}$.

Fig. 12 shows the initial condition specified at $t=0$ along with the variation in the $\varphi$ profile with time. The initial sine wave pattern transforms into the pattern shown in Fig. 12. This pattern remains stationary, as determined from numerical simulation, and does not change its form as time evolves. Fig. 13 shows the $\varphi_{t}$ evolution for this simulation. The zero values of $\varphi_{t}$ along the domain are consistent with stationary $\varphi$ profile.

Fig. 14 shows the plot of $\varphi_{x}$ for the simulation representing dislocation microstructure. The $\varphi_{x}$ initially in the form of a cosine pattern evolves into the 
pattern shown in the figure. The pattern has both positive and negative dislocations co-existing in a quasi-equilibrium state under no load. Fig. 15 shows the stress profile of the dislocation microstructure. High stresses are observed in the dislocation core; however, these are short-ranged and decrease rapidly away from the core. Due to the short-range nature of these stresses there is no interaction between the adjacent dislocation walls at zero loads unless they are very close and hence the positive and negative dislocation walls do not attract and coalesce. Likewise, the two positive dislocation walls do not repel each other and hence the shown micro-structure is retained in a quasi-equilibrium state. This behavior is different from that observed in Sec. 2.3 where the profiles corresponding to two dislocation walls of the same sign adjacent to each other were not equilibria. When loads are applied, the dislocation walls move under the influence of loads leading to interaction between adjacent neighbors and leading to eventual annihilation of the entire microstructure. This phenomenon is discussed in Section (4.2).

\subsection{Multiple dislocation walls subjected to loading}

To observe the response under time varying loads, the initial condition in the form of sine waves described before is imposed on $\varphi$. The sample is then loaded by uniformly increasing the applied strain, $g$, from 0 to 0.01 .

A plot of the spatial average of $\tau$ over the entire domain vs. $g$, which can be interpreted as a stress-strain curve is shown in Fig. 16. A section from the sample is observed which is shown in Fig. 16 (a). As the applied strain starts to increase the sine waves evolve into a step-like pattern shown in Fig. 16 (b). These steps remain stationary or move with very slow velocity until the applied strain increases to a particular value. This phase in the stress strain curve can be interpreted as an initial elastic phase. When applied strain becomes more than 0.0005 as in Fig. 16 (c) steps having positive magnitude start to move with higher velocities and coalesce with adjacent steps. At $g=0.0015$ all the positive magnitude steps coalesce to form the pattern shown in Fig. 16 (d). 
This phase, from $g=0.0005$ to $g=0.0015$, can be interpreted as a plastic phase since the stress remains constant or decreases slightly with increasing strain. As applied strain increases from 0.0015 to 0.003 , another elastic phase is observed in which the steps in Fig. 16 (d) remain stationary or move very slowly. This is followed by a plastic phase from $g=0.003$ to $g=0.004$ during which the steps indicated in Fig. 16 (d) coalesce and result in the pattern shown in Fig. 16 (e). After this there is no elastic phase, the remaining steps continue to move and the velocity increases to larger values as $g$ increases. This results in a drop in the stress strain curve until the point where all steps eventually collapse producing a homogenous equilibrium condition shown in Fig. 16 (f). A further increase in $g$ produces a response similar to the elastic curve.

\subsection{Rate dependent behavior}

The response to different rates of loading is shown in Fig. 17. The multiple sine wave initial condition explained earlier is imposed on $\varphi$ and the applied strain, $g$ , is increased from 0 to 0.01 at different rates. The stress strain response for these simulations exhibits rate-dependent behavior. For the case in which the applied strain rate is higher, the elastic and plastic phases are comparatively longer as compared to the case in which the applied strain rate is lower. For the case with higher applied strain rate, the positive and the negative dislocations coalesced at values of load higher than in the case with lower applied strain rate.

\subsection{Stick-slip behavior in the average stress-strain response}

Fig. 18 illustrates the stick slip behavior observed when multiple dislocation walls are subjected to loading. Multiple sine waves described above are prescribed as an initial condition for $\varphi$. The sample is loaded quasi-statically by imposing a constant load for 20 time units. For each constant load, the average of $\varphi$ over the entire domain is computed at the end of the loading sequence as follows: 


$$
\operatorname{avg} \varphi=\frac{\sum_{h=1}^{N} \varphi^{k}\left(x_{h}\right)}{N},
$$

where $\mathrm{N}$ is the total number of nodes and $x_{h}$ is the position of the $h^{\text {th }}$ node. The absolute value of average $\varphi$ is normalized by the constant $\bar{\varphi}$ to compute $\varphi^{*}$.

$$
\varphi^{*}=\frac{|\operatorname{avg} \varphi|}{\bar{\varphi}}
$$

$\varphi^{*}$ is plotted versus the constant load as shown in Fig. 18. The average of $\varphi$ can be interpreted as average plastic strain. For small loads, the average plastic strain is close to zero. After a threshold value of load there is a sudden increase in the average plastic strain.

\section{Idealized Fatigue Microstructure}

The motivation for the simulations discussed in this section comes from the persistent slip band phenomenon observed in fatigue experiments in Ahmed Wilkinson and Roberts (1997) and Mughrabi (1979) as shown in Fig. 19, which is a hand-sketch of the original picture. To emulate the persistent slip band structure the following initial condition for $\varphi$ is prescribed. A repeated sequence of sets, with each set consisting of three steps is specified. All steps have the same width and spacing, and magnitude equal to $\bar{\varphi}$. Each set of steps is separated by a wide gap in between. The evolution of $\varphi$ under no load is analyzed. Fig. 20 shows the idealized fatigue microstructure obtained from the simulation. The sharp corners of the steps become smooth instantaneously. The steps do not evolve appreciably (noticeably) with time after they attain the smooth profile. Fig. 21 is the plot of $\varphi_{x}$ for a section of the sample and can be interpreted as microstructures with the peaks representing dislocation walls. Under no load, dislocation cell-wall microstructures exist in a quasi-equilibrium state. Fig. 22 represents the stresses in these dislocation walls. High stresses are observed in the dislocation cores. These stresses decrease rapidly away from the cores. 


\section{Significance of transport represented by leading $\left|\varphi_{x}\right|$ term in (1)}

The significance of the leading $\left|\varphi_{x}\right|$ term in the governing equation (1) is discussed in this section. A comparison of the results of the tests performed with zero loads illustrates that the results of the computation are same irrespective of whether the leading term in the governing equation is $\left|\varphi_{x}\right|$ or 1 . One might then question the need to have the leading $\varphi_{x}$ term in the governing equation. Certainly, the absence of the leading $\varphi_{x}$ term would simplify the physical and numerical understanding of the governing equation as well as ease the complexity of the solution procedure. However, important differences are observed, both when loads are applied and they are absent. Consider the case shown in Fig. 23 (a) where a tanh profile with the center at $x=500 \mathrm{~b}$ is prescribed as an initial condition on $\varphi$. The value of $g$ is increased linearly from 0 to 0.01 to model a linear ramp strain loading. The corresponding $\varphi_{x}$ plot is shown in Fig. 23 (b). For

the $F=\left|\varphi_{x}\right|$ case, the $\varphi$ profile moves towards the right with increase in loads as discussed earlier in section 3.1. However, for the $F=1$ case (the Ginzburg Landau approximation for the given energy), there is no lateral movement in the $\varphi$ profile and the dislocation does not move under the application of loads. The $\varphi$ profile simply shifts upwards by the value of the applied load $g$. After each increment of load the profile jumps from the existing equilibrium configuration to the corresponding equilibrium configuration of the higher load. Numerically, the lack of dislocation motion can be explained by the calculation that the velocity $c$ (section 1.3) is zero when the leading $\varphi_{x}$ term is absent. Thus, at least in this restricted setting, the GL equations (for the assumed energy) cannot model dislocation motion under applied load, while our model does. Of course, representing dislocation motion under applied load has to be an essential ingredient of any model of dislocation dynamics and plasticity.

Differences are also observed between the results of the simulations when the specified initial condition (IC) has transition layers between wells as shown in 
Fig. 24 (a). A comparison is made between the simulations performed with the GL equations associated with the FDM model. This GL equations is obtained by replacing the leading $\left|\varphi_{x}\right|$ term in the governing equation (1) to 1 . The simulations are performed with $g$ set to zero. For the GL simulation, the initial $\varphi$ profile is not a (quasi)equilibrium. The profile shifts to a form where the transition layers connect the wells. After this form is attained, the $\varphi$ profile remains stationary. Since the overall profile is not periodic in space, we conclude that the final 'state' is a quasi-equilibrium.

In the case of FDM, there is a minor adjustment from the initial condition and then the $\varphi$ profile remains practically unchanged in time Fig. 24 (a). A section of the $\varphi$ profile showing only a single transition layer is shown in Fig. 24 (b). The section of the $\varphi_{x x}$ profile is shown in Fig. 24 (c). The results in AMZ (2010) indicate that in FDM it is possible to have spatially non-periodic equilibria, where fronts connect levels between energy wells. Thus an interesting question arises as to whether the observed profiles as in Fig. 24(a,b) are strict equilibria of FDM, or that the class of quasi-equilibria of FDM is larger than that admitted in the corresponding GL equations. To test this hypothesis, we utilize a result of AMZ (2010) that suggests that strict equilibrium transition layers in FDM connecting levels in between energy wells necessarily have discontinuities in their $\varphi_{x x}$ profile. Figs. 24(c) indicates no such discontinuity. This suggests that the FDM $\varphi$ profile of Fig. 24 (a) is in a quasi-equilibrium state. This is further verified by exploring the $\varphi_{x x}$ profile for a definite quasi-equlibrium in FDM, as shown in Fig. 25(a,b,c). Here, the levels of the transition layers are very close to the energy wells. At the end of the simulation there is negligible change in the initial $\varphi$ profile. A section of $\varphi$ and $\varphi_{x x}$ profiles is shown in Fig. 25 (b) and (c), respectively. The levels of the transition layers remain stationary even when they are very close to the energy wells. The $\varphi_{x x}$ profile is also continuous and qualitatively similar to that of Fig. 24(c). 


\section{Numerics}

\subsection{Significance of the diffusion term}

The significance of the diffusion term $\varepsilon \varphi_{x x}$, arising from the modeling of core energy, is discussed in this section. The $\varphi_{x x}$ plot corresponding to the initial condition on a small section of the sample is shown in Fig. 26 (a). A simulation is performed with $\varepsilon$ set to zero so that there is no contribution from the diffusion term. In the absence of the diffusion term, kinks develops in the $\varphi_{x x}$ values as shown in Fig. 26 (b) which become sharper with time as shown in Fig. 26 (c) and (d). A slightly larger section is shown in Fig. 27 to observe the effect of $\varepsilon$ set to zero. The smooth transition layers in $\varphi$ shown in Fig. 27 (a) are transformed in to a step-like form shown in Fig. 27 (b). In the presence of the diffusion term, kinks do not develop in the $\varphi_{x x}$ values and the smooth transition layers in $\varphi$ are maintained.

\subsection{Spikes in $\varphi_{t}$ profile}

The influence of a modified numerical scheme on the results obtained from the simulation of the governing equation is discussed in this section. The update of $\varphi$ given by (10) is modified as follows to incorporate the Friedrichs scheme.

$$
\begin{aligned}
& \text { if } c^{k}\left(x_{h}\right) \neq 0 \\
& \begin{array}{l}
\varphi^{k+1}\left(x_{h}\right)=\varphi^{k}\left(x_{h}\right)+\varphi_{t}^{k}\left(x_{h}\right) \Delta t^{k} \\
\text { if } c^{k}\left(x_{h}\right)=0 \quad \text { Lax Friedrichs } \\
\varphi^{k+1}\left(x_{h}\right)=\frac{\varphi^{k}\left(x_{h+1}\right)+\varphi^{k}\left(x_{h-1}\right)}{2}+\varphi_{t}^{k}\left(x_{h}\right) \Delta t^{k} .
\end{array}
\end{aligned}
$$


The simulation discussed in section 4 is performed with the above modified numerical scheme. Figs. 28 and 29 are the $\varphi$ and $\varphi_{t}$ profile, respectively, for the simulation performed with Friedrichs scheme. They can be compared with Figs. 12 and 13 which are the corresponding plots obtained from the simulation performed without the Friedrichs scheme. There is no noticeable difference in the trend of $\varphi$ evolution between Figs. 12 and 28. However, from the comparison of the $\varphi_{t}$ evolution plots between Figs. 13 and 29, spikes are observed in the case with the Friedrichs scheme.

The occurrence of spikes in the $\varphi_{t}$ plot shown in Fig. 29 is further discussed in this section. The spikes occur in the region where the $\varphi$ values coincide with the points in the energy curve where equilibrium is unstable. A small section of the domain is analyzed in Fig. 30 that illustrates the sequence of occurrence of a single spike. Fig. 30 (a) shows the $\varphi_{x x}$ values in the section of the sample at the instant of time just before the occurrence of the spike. As time progresses, kinks develop in the $\varphi_{x x}$ values as shown in Fig. 30 (b). Since there is no change in value of $\tau$ as the value of $g$ is constant and the change in the value of $\varphi$ is negligible, a kink in $\varphi_{x x}$ values results in an increase in the value of $\varphi_{t}$ which corresponds to a spike in the $\varphi_{t}$ plot. These kinks then smooth out with time as shown in Fig. 30 (c) and (d) resulting in the original smooth $\varphi_{x x}$. This phenomenon keeps on continuing at the same point in space but at random instants of time resulting in more spikes. The height of the spikes varies in space as well as time. It is important to note that without the incorporation of Friedrichs scheme in numerical calculation discussed in (14) there is no kink in $\varphi_{x x}$ values and the spikes are absent. Thus, the intermittency appears to be a result of using the Friedrichs scheme and whether this is consistent with the PDE needs to be determined.

\section{Convergence}


The effect of mesh refinement on the results obtained by the model is discussed in this section. For the sake of convenience, a convergence study is shown for only one of the setups. The setup described in section 4 to study the behavior of multiple dislocation walls under no load is used for the study. Simulations are performed with three meshes of 2000, 4000 and 8000 elements. Fig. 31 shows the effect of refinement on the $\varphi$ and $\varphi_{x}$ profiles. A good convergence in both $\varphi$ as well as $\varphi_{x}$ profile is observed as there is negligible difference between the profiles obtained with different meshes. To quantify the differences in the results for different meshes the following error norms are computed.

$$
\begin{aligned}
& \xi(\varphi)^{4000-2000}=\frac{\sqrt{\int_{L}\left(\varphi^{4000}-\varphi^{2000}\right)^{2} d x}}{\frac{1}{2}\left(\sqrt{\int_{L}\left(\varphi^{4000}\right)^{2} d x}+\sqrt{\int_{L}\left(\varphi^{2000}\right)^{2} d x}\right)} \\
& \xi(\varphi)^{8000-4000}=\frac{\sqrt{\int_{L}\left(\varphi^{8000}-\varphi^{4000}\right)^{2} d x}}{\frac{1}{2}\left(\sqrt{\int_{L}\left(\varphi^{8000}\right)^{2} d x}+\sqrt{\int_{L}\left(\varphi^{4000}\right)^{2} d x}\right)},
\end{aligned}
$$

where $\varphi^{2000}, \varphi^{4000}$ and $\varphi^{8000}$ denote the $\varphi$ values obtained using a mesh of 2000, 4000 and 8000 elements, respectively. $\xi(\varphi)^{4000-2000}$ denotes the error norm for comparison between $\varphi$ values for meshes of 4000 and 2000 elements. Likewise, $\xi(\varphi)^{8000-4000}$ is the error norm for comparison between $\varphi$ values for meshes of 8000 and 4000 elements. The corresponding error norms for $\varphi_{x}$ values are obtained from

$$
\begin{aligned}
& \xi\left(\varphi_{x}\right)^{4000-2000}=\frac{\sqrt{\int_{L}\left(\varphi_{x}^{4000}-\varphi_{x}^{2000}\right)^{2} d x}}{\frac{1}{2}\left(\sqrt{\int_{L}\left(\varphi_{x}^{4000}\right)^{2} d x}+\sqrt{\int_{L}\left(\varphi_{x}^{2000}\right)^{2} d x}\right)} \\
& \xi\left(\varphi_{x}\right)^{8000-4000}=\frac{\sqrt{\int_{L}\left(\varphi_{x}^{8000}-\varphi_{x}^{4000}\right)^{2} d x}}{\frac{1}{2}\left(\sqrt{\int_{L}\left(\varphi_{x}^{8000}\right)^{2} d x}+\sqrt{\int_{L}\left(\varphi_{x}^{4000}\right)^{2} d x}\right)},
\end{aligned}
$$


where $\varphi_{x}^{2000}, \varphi_{x}^{4000}$ and $\varphi_{x}^{8000}$ denote the $\varphi_{x}$ values obtained using a mesh of 2000, 4000 and 8000 elements, respectively. The variation of error norms with time is shown in Fig. 32. The magnitude of error norms does not change appreciably with time. The magnitude of $\xi(\varphi)^{8000-4000}$ is less than that of $\xi(\varphi)^{4000-2000}$. The magnitude of $\xi\left(\varphi_{x}\right)^{8000-4000}$ is also less than that of $\xi\left(\varphi_{x}\right)^{4000-2000}$. This suggests that the results obtained from the simulations are converging with mesh refinement.

\section{Conclusions}

We have been able to demonstrate the following phenomena.

- Our primary result is the demonstration of spatially non-periodic patterned microstructure, indistinguishable from being stationary in time for all practical purposes, but non-predictable from the equilibrium equations of the model. We term such states quasi-equilibria.

- The model is capable of predicting motion of dislocation walls as localized entities (walls being the only possibility in 1-d). The average velocity curve of such a localized profile resembling a dislocation wall starts with a zero slope for small strains and then varies approximately as square root of the applied strain at larger strains.

- Patterned plastic deformation fields are shown to be in quasi-static equilibrium under no loads.

- Dislocation cell wall microstructures with features common to the persistent slip band phenomenon of fatigue experiments are shown to be quasi-equilibrium microstructure of the model.

- Elasto-plastic behavior is observed when simulations are performed with non-zero loads. The stress strain response is elastic at small loads and transforms to plastic after a load threshold is exceeded, characterizing the phenomenon of yielding. The stress strain curve exhibits rate-dependent behavior.

- Stick-slip behavior in average equivalent plastic strain is also predicted. 
An interesting case of intermittency, possibly a numerical artifact, is observed when the Friedrichs scheme is incorporated. The use of Friedrichs scheme may be debated as a similar plastic deformation field is observed without the intermittent behavior when Friedrichs scheme is omitted. However, given the current interest in models predicting intermittency, it seems that the Friedrichs scheme can be a useful recipe that can be translated into a nonlocal physical model.

\section{Acknowledgement}

This work was partially funded by the National Science Foundation through CMU MRSEC, grant no. DMR-0520425.

\section{References}

Acharya, A., 2010, New inroads in an old subject: plasticity, from around the atomic to macroscopic scale, Journal of the Mechanics and Physics of Solids, 58, 766- 778.

Acharya, A., Matthies, K., Zimmer, J., 2010, Wave solutions for a quasilinear model of field dislocation mechanics, Journal of the Mechanics and Physics of Solids, 58, 2043-2053.

Acharya, A. Tang, H., Saigal, S., Bassani, J. L. 2004, On boundary conditions and plastic straingradient discontinuity in lower-order gradient plasticity, Journal of the Mechanics and Physics of Solids, 52, 1793-1826.

Acharya, A. and Tartar, L., 2011, On an equation from the theory of field dislocation mechanics, Bulletin of the Italian Mathematical Union, 9(IV), 409-444.

Ahmed, J., Wilkinson, A. J., Roberts, S. G., 1997, Characterizing dislocation structures in bulk fatigued copper single crystals using electron channeling contrast imaging (ECCI), Philosophical Magazine Letters, 76, 237-245

Carr, J. and Pego, R. L., 1989, Metastable Patterns in solutions of $u_{t}=\varepsilon^{2} u_{x x}-f(u)$, Communications on Pure and Applied Mathematics, 42, 523-576 
Mughrabi, H., Ackermann, F., Herz, K., 1979, Persistent slip bands in fatigued face centered and body centered cubic metals, Technical Report, American Society for Testing and Materials,

Philadelphia, 69-105 


\section{Figures}
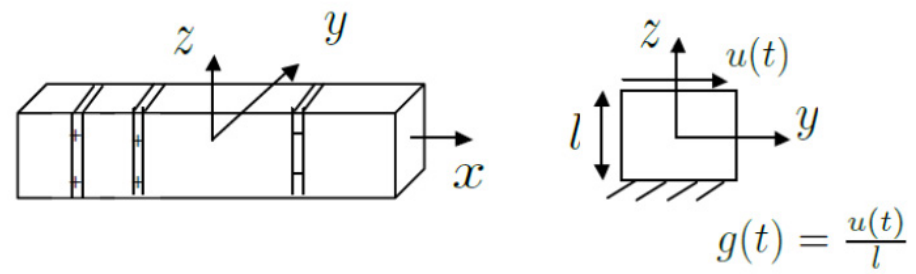

Fig. 1 Infinite rectangular cylinder subjected to simple shear 


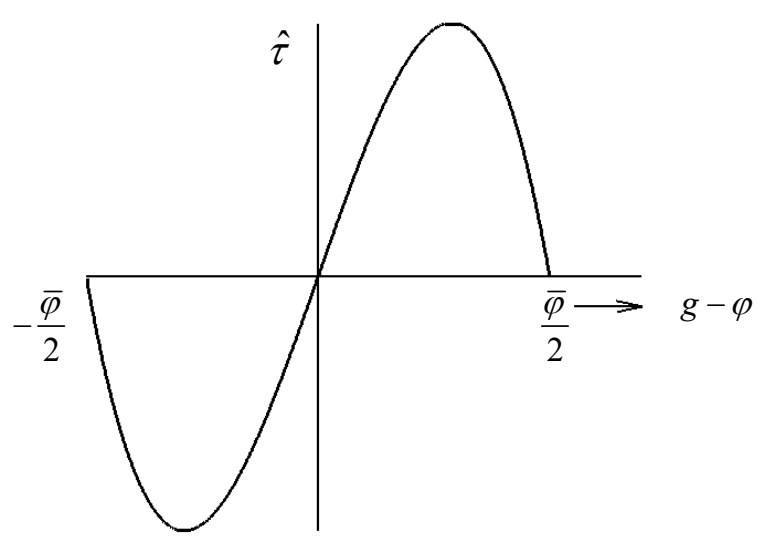

Fig. 2 (a) Plot of function $\hat{\tau}$

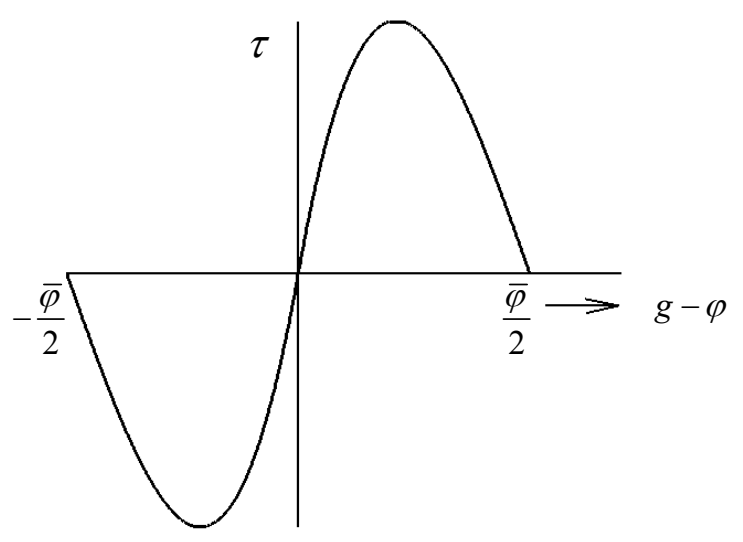

Fig. 2 (b) Plot of function $\tau$ 


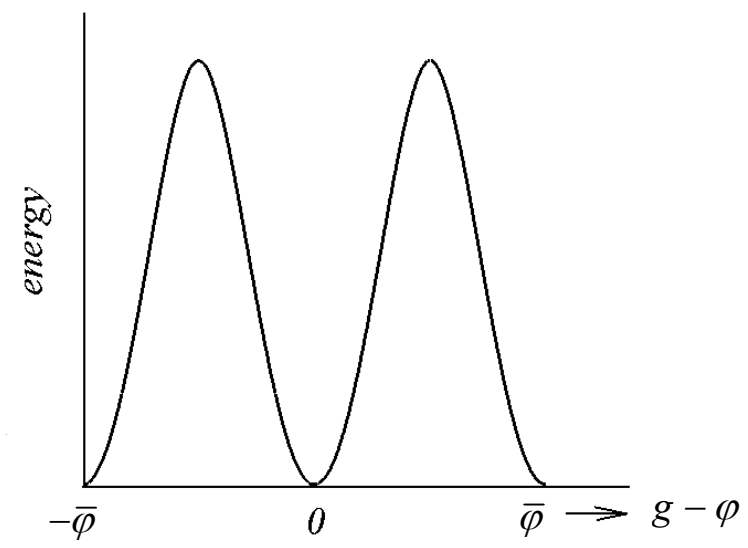

Fig. 2 (c) Plot of energy corresponding to the stress function $\hat{\tau}$ 


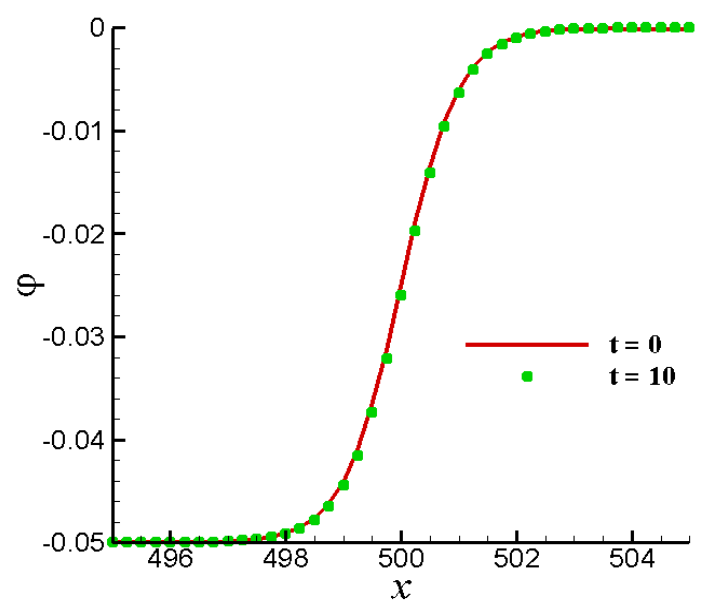

Fig. 3 tanh equilibrium profile 


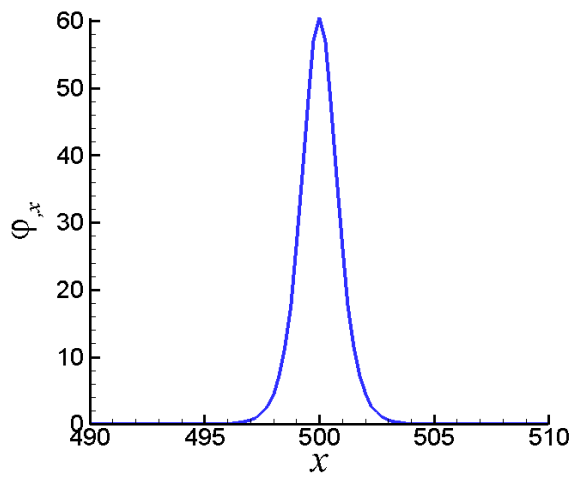

Fig. 4 (a) Screw dislocation wall

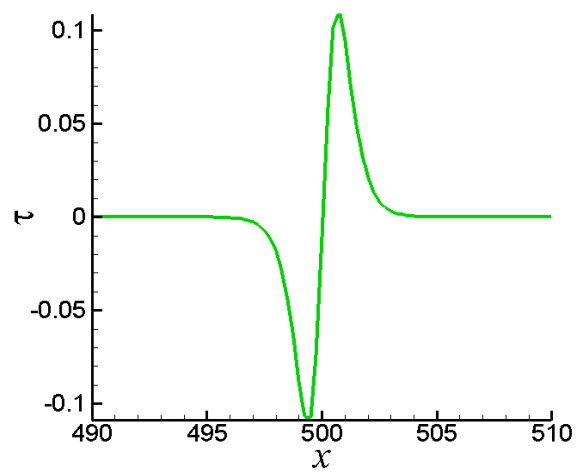

Fig. 4 (b) Stress in a dislocation wall 


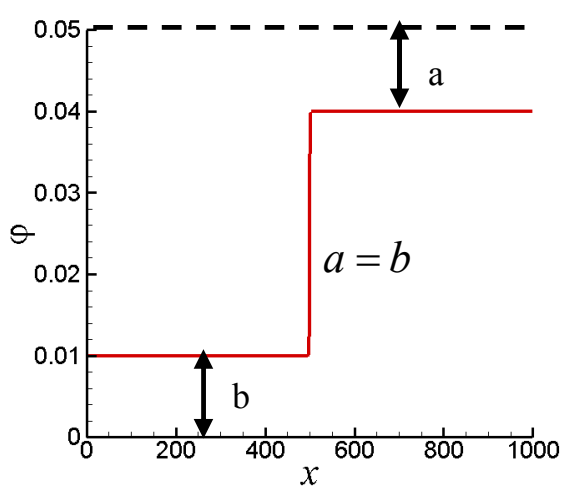

$$
\mathrm{t}=0
$$

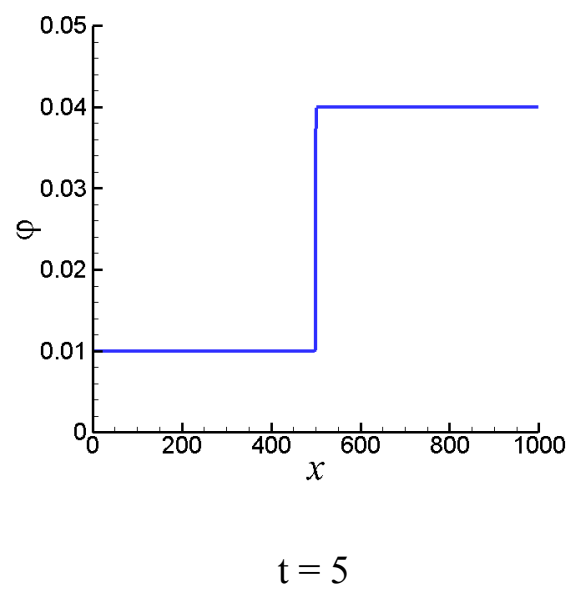

Fig. 5 Transition layer equidistant from energy wells 


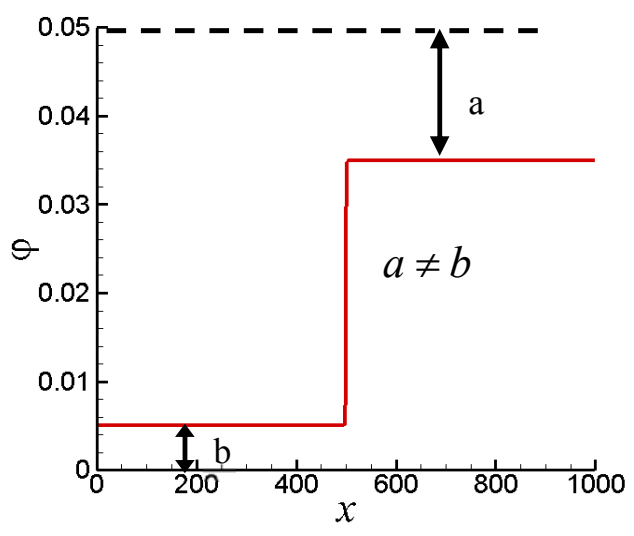

$t=0$

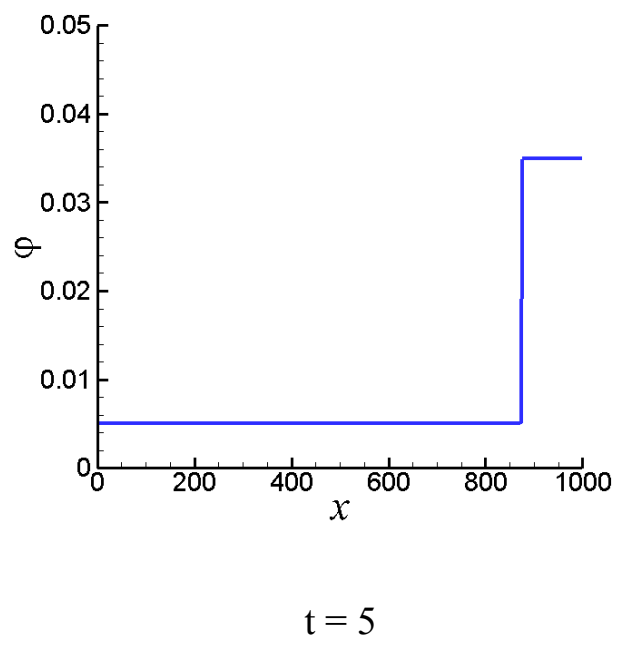

Fig. 6 Non-equilibrium transition layer non-equidistant from energy wells 

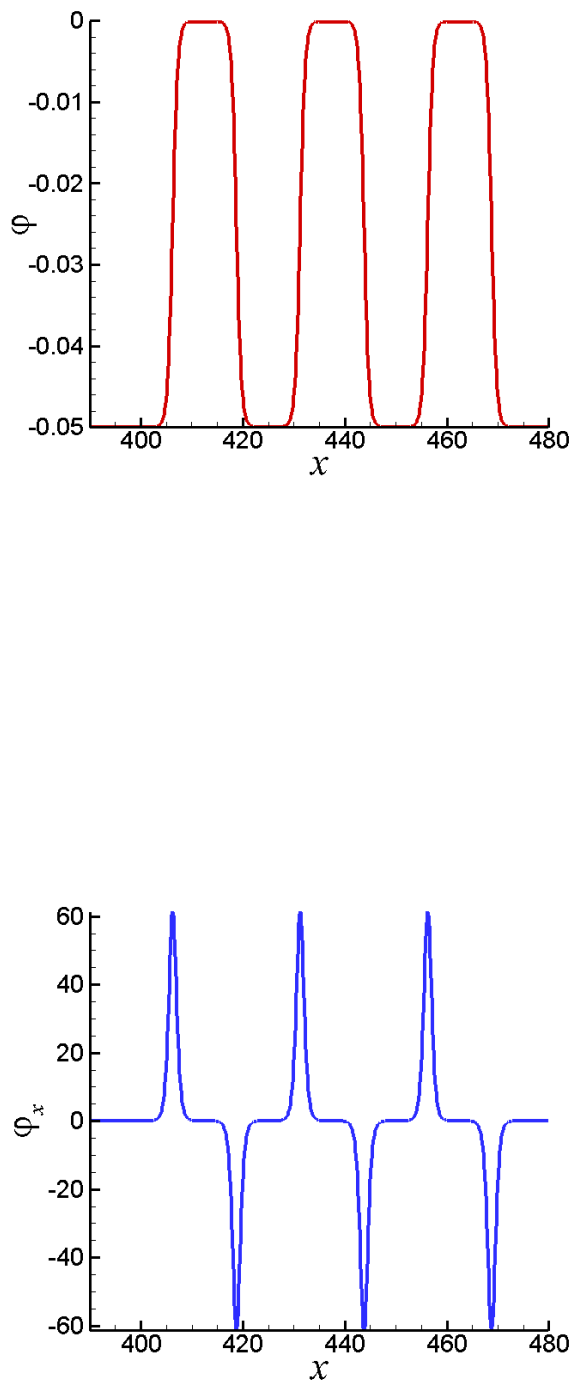

Fig. 7 Dipolar Structure: Quasi-equilibrium transition layers connecting wells corresponding to positive and negative dislocations 

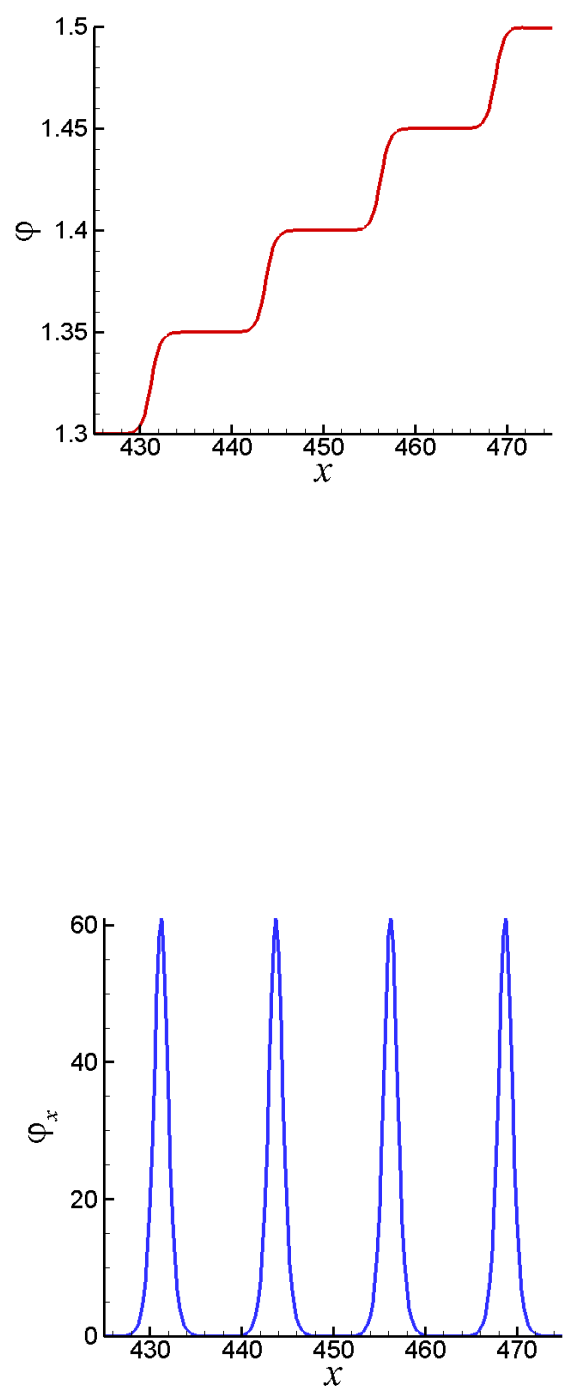

Fig. 8 Pile-up: Quasi-equilibrium transition layers connecting wells corresponding to only positive dislocations 

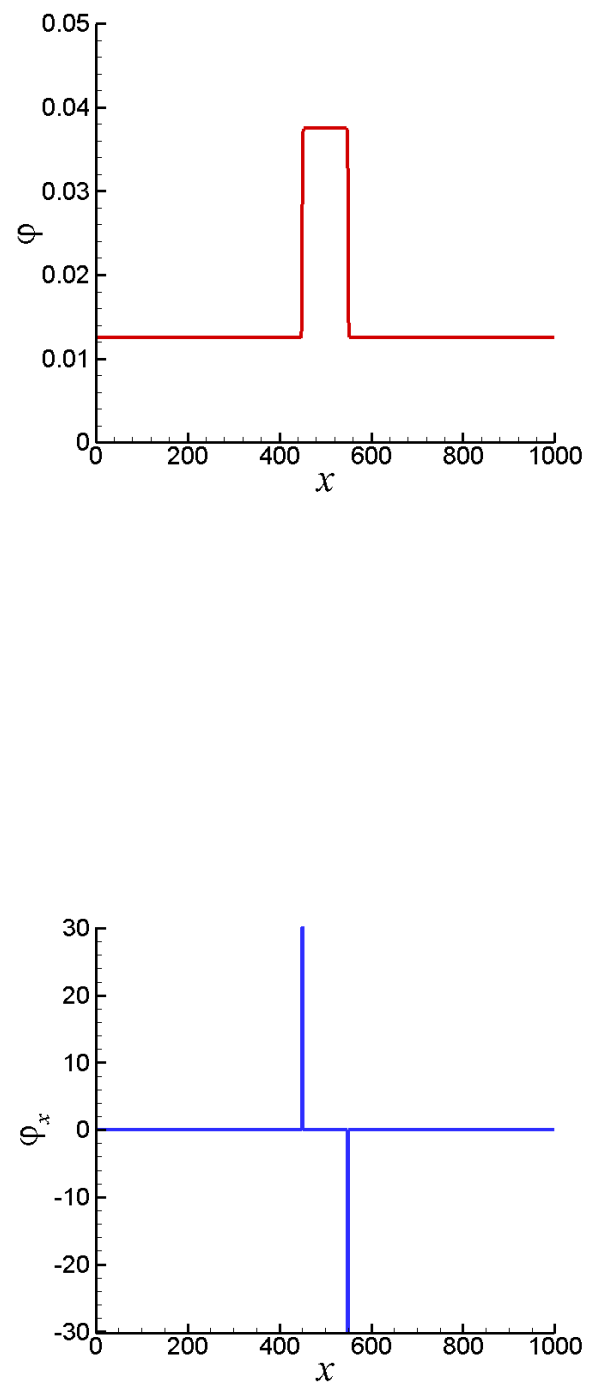

Fig. 9 Transition layers between wells corresponding to positive and negative dislocation 

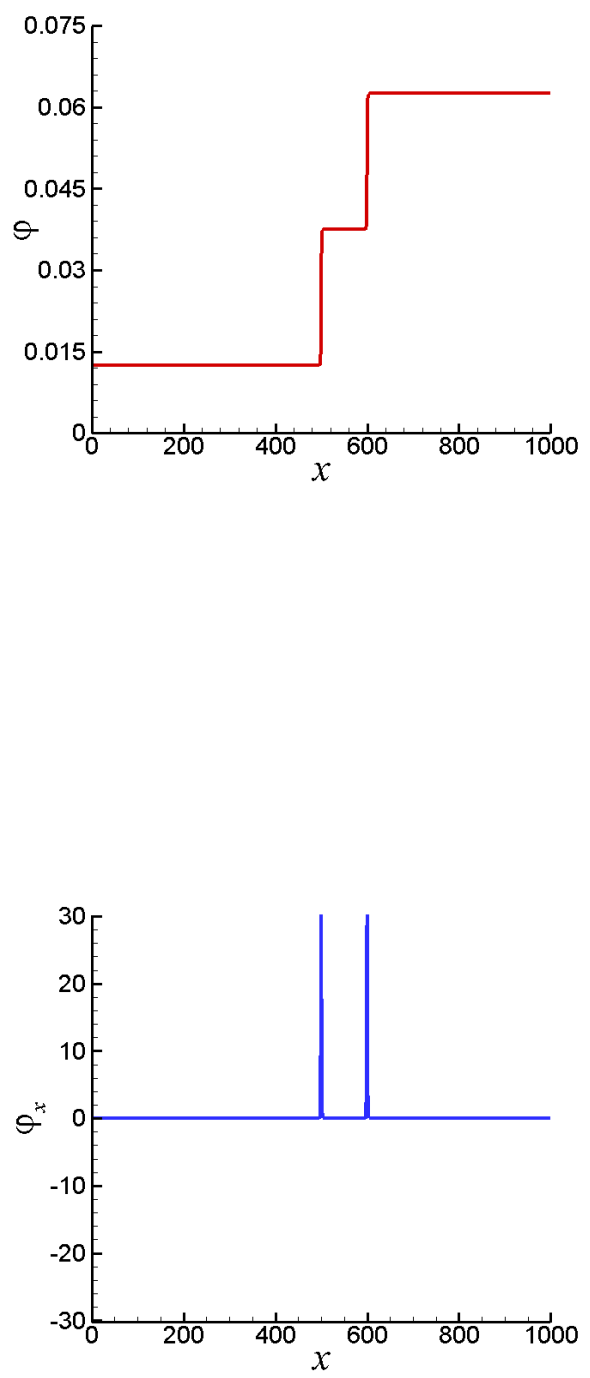

Fig. 10 Non-equilibrium transition layers between wells corresponding to only positive dislocations 

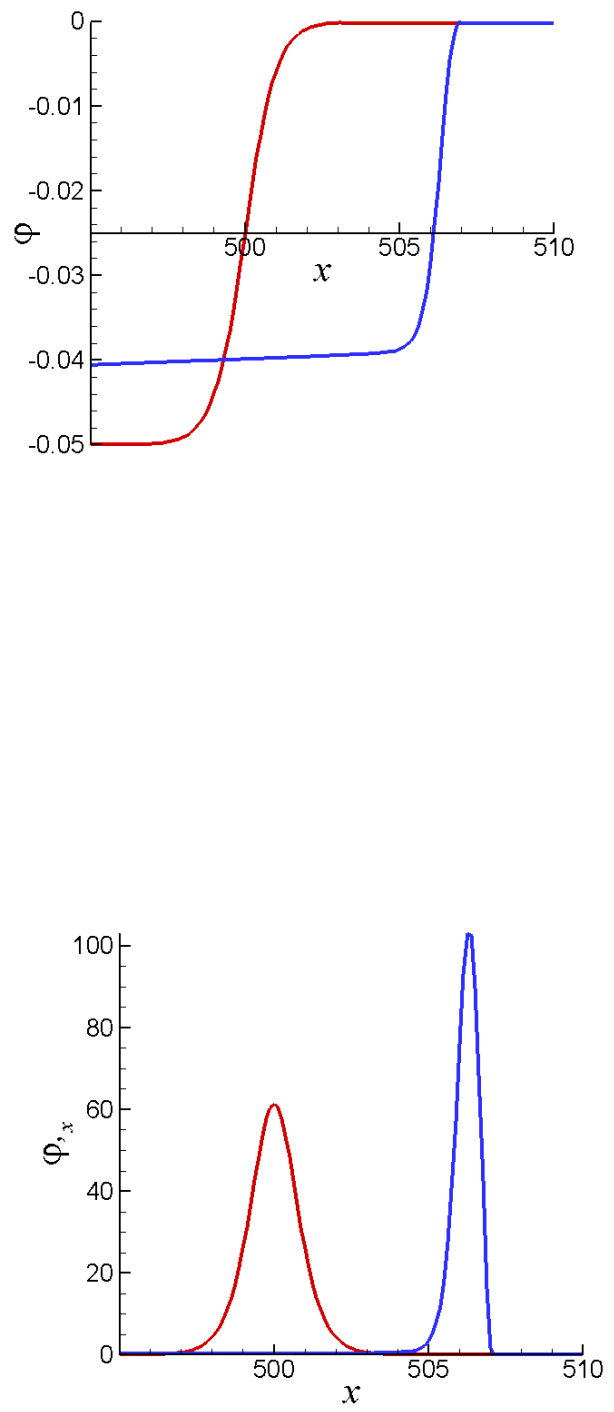

Fig.11 (a) Dislocation motion 


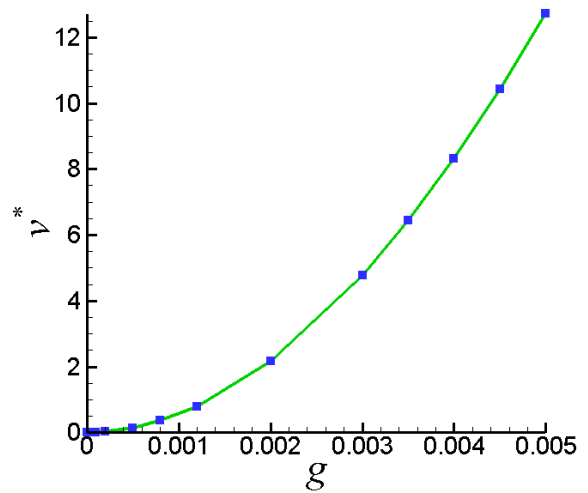

Fig. 11 (b) Dislocation velocity vs. strain 


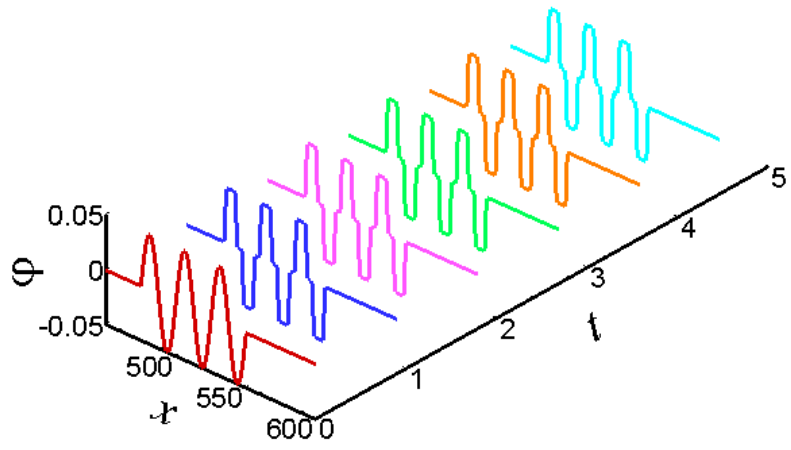

Fig. 12 Plot of variation of $\varphi$ for multiple sine wave initial condition

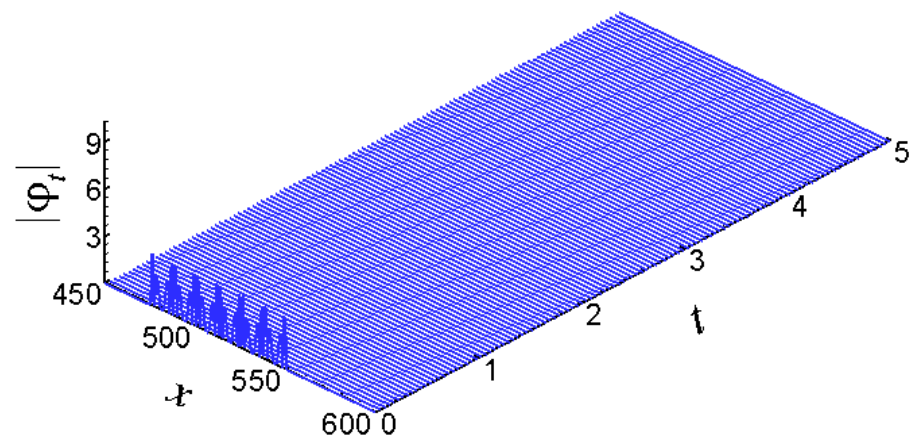

Fig. $13 \varphi_{t}$ plot for multiple sine wave initial condition 


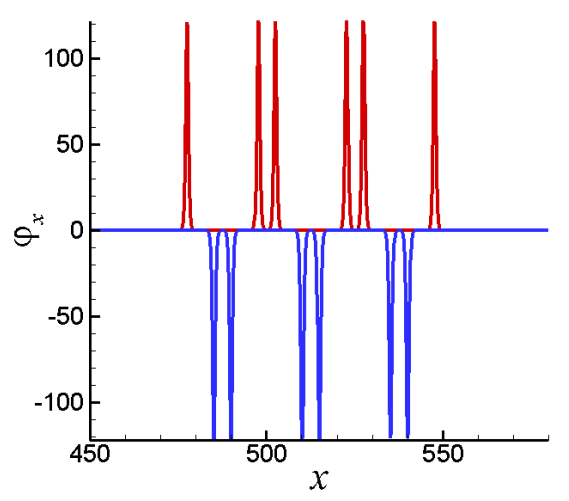

Fig. 14 Quasi-Equilibrium dislocation microstructure

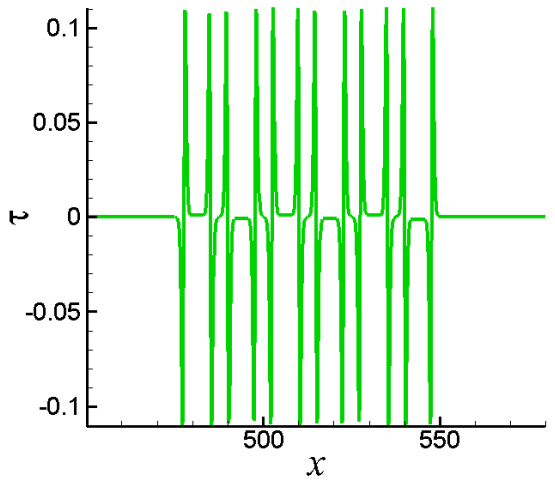

Fig. 15 Dislocation stress profile 


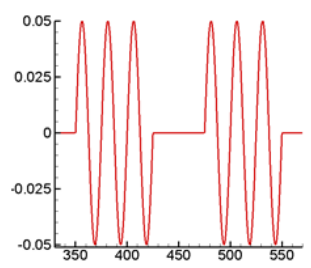

(a) initial condition

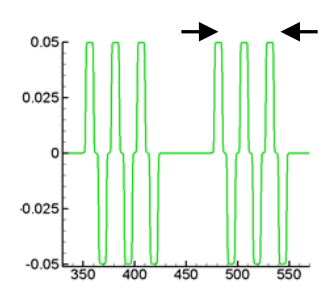

(b) $g=0$

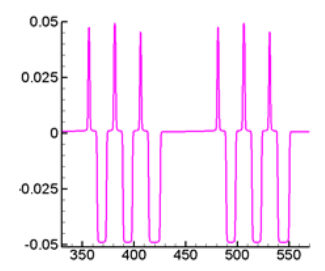

(c) $g=0.0015$

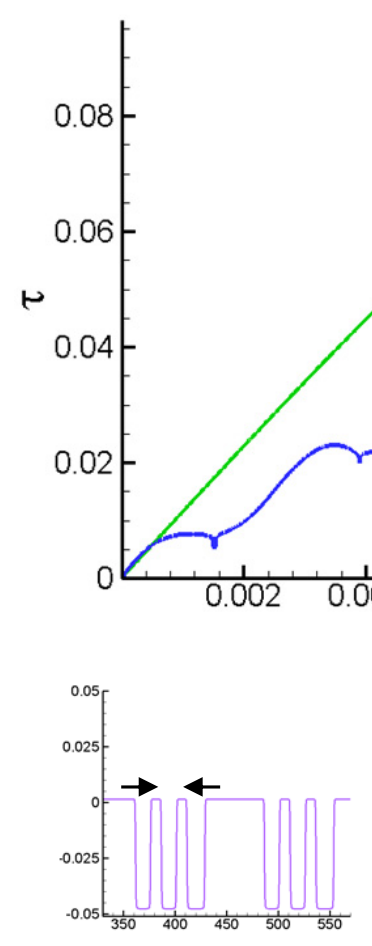

(d) $g=0.003$

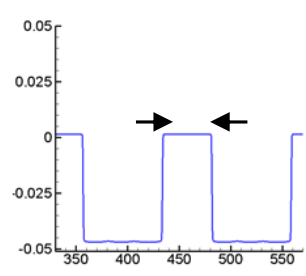

(e) $g=0.004$

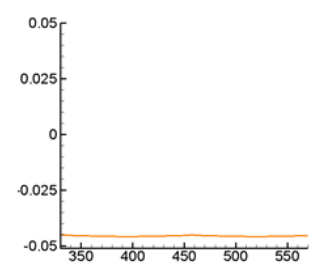

(f) $g>0.0052$

Fig. 16 Multiple dislocation walls subjected to loading 


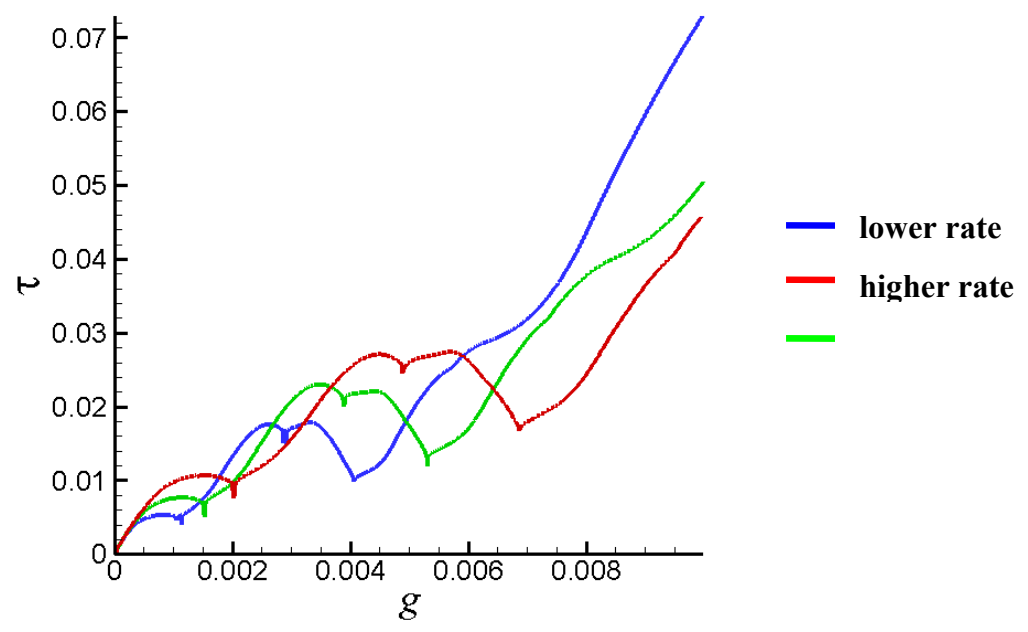

Fig. 17 Multiple dislocation walls subjected to different rates of loading 


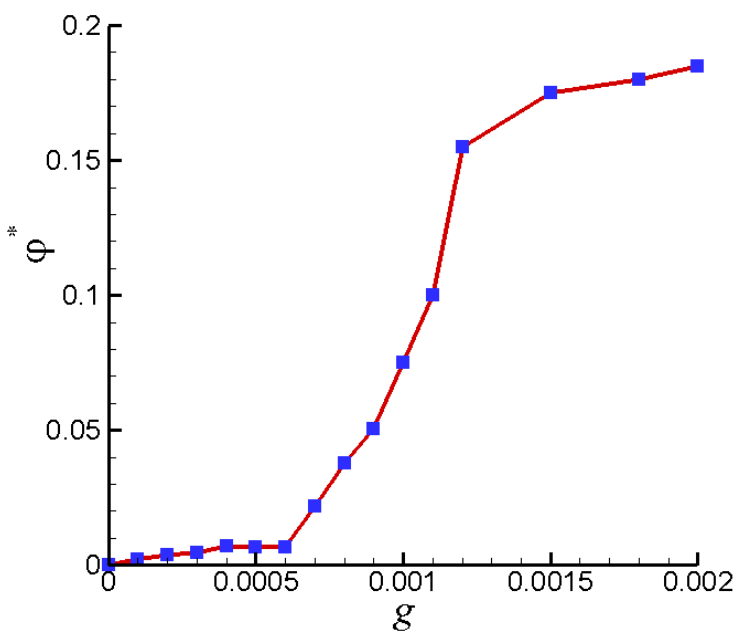

Fig. 18 Stick-slip behavior 


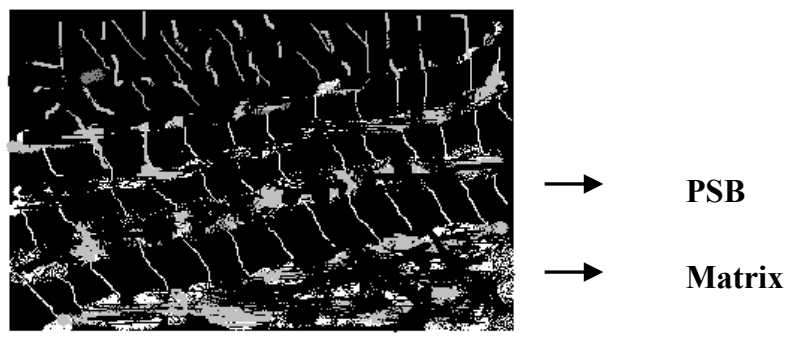

Fig. 19 Hand-sketch of Fatigue Microstructure picture originally shown in Ahmed Wilkinson and Roberts (1997)

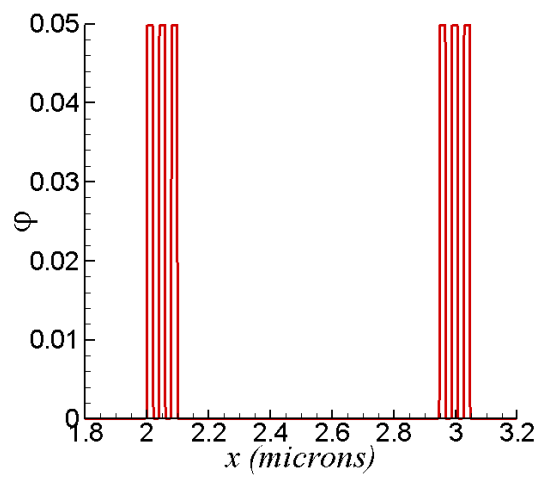

Fig. 20 Idealized fatigue cell wall microstructure obtained from simulation 


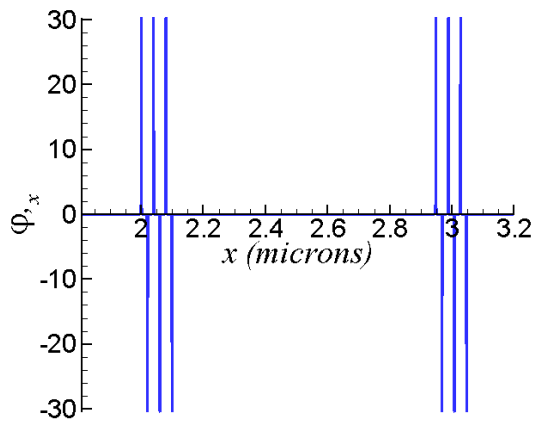

Fig. 21 Dislocation cell-wall microstructure

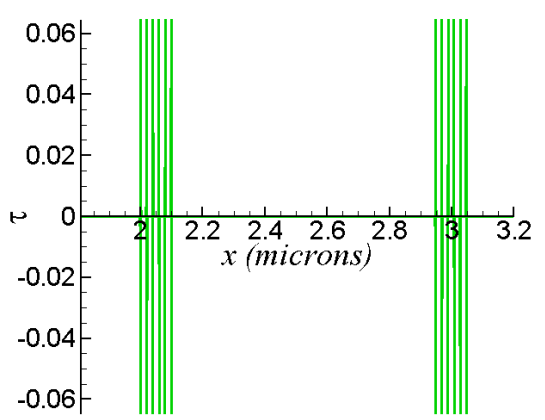

Fig. 22 Dislocation stress profile 


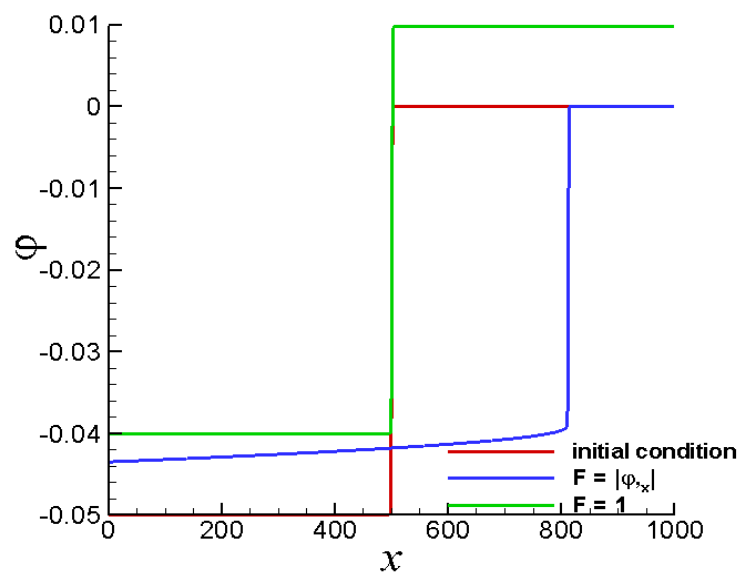

(a) $\varphi$ plot

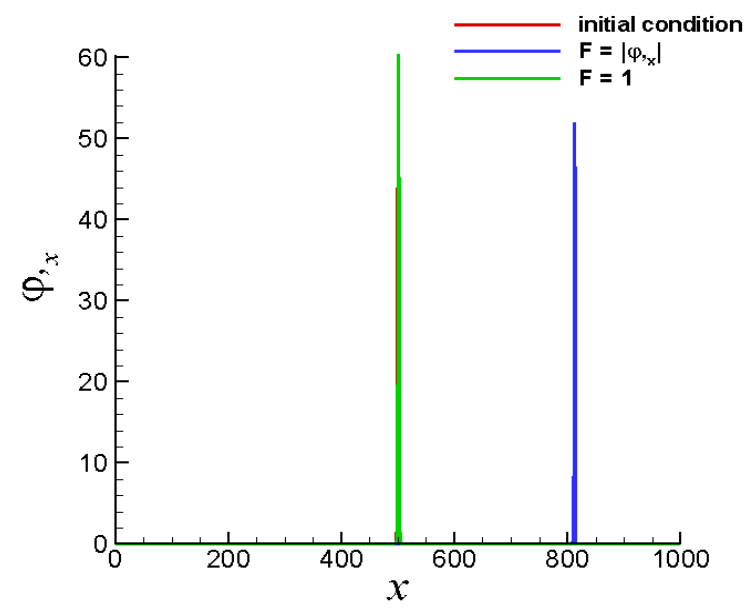

(b) $\varphi_{x}$ plot

Fig. 23 Influence of leading $\varphi_{x}$ term in the governing equation (single transition layer connecting wells) 


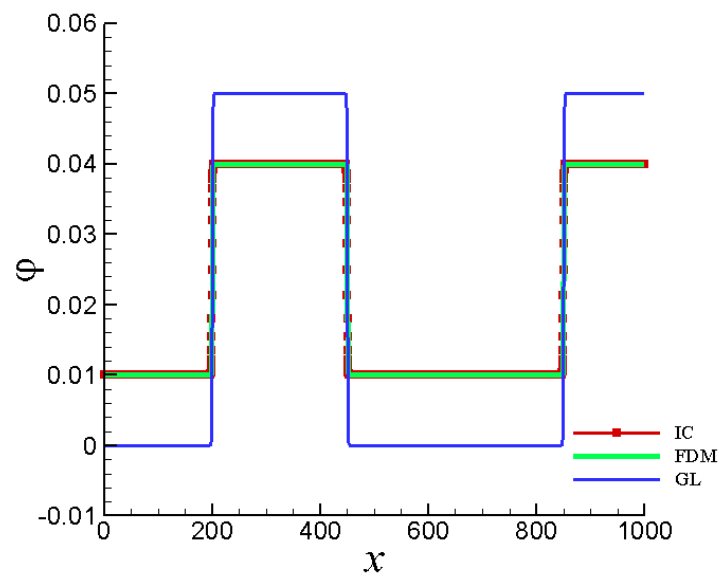

Fig. 24 (a) Influence of leading $\varphi_{x}$ term in the governing equation (multiple transition layers between wells) 


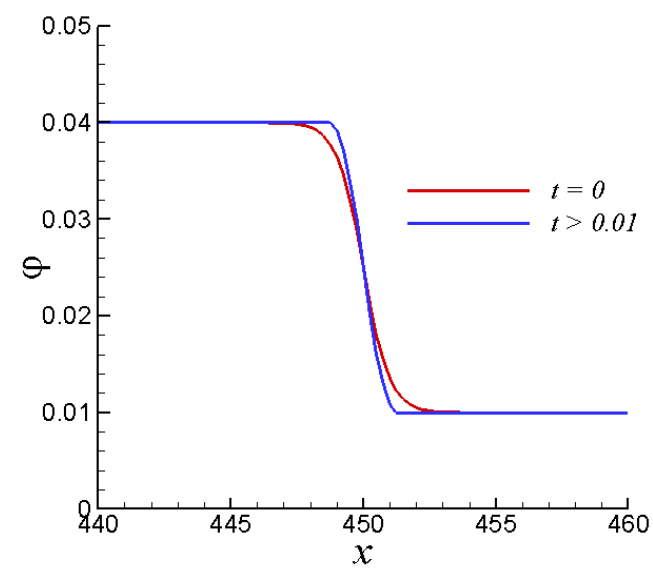

Fig. 24 (b) A section of the $\varphi$ profile obtained from the FDM model showing a single transition layer

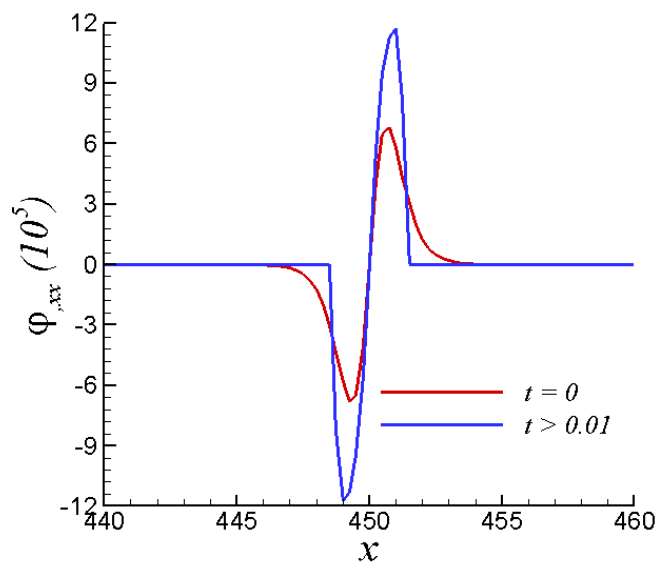

Fig. 24 (c) $\varphi_{x x}$ profile across a transition layer 


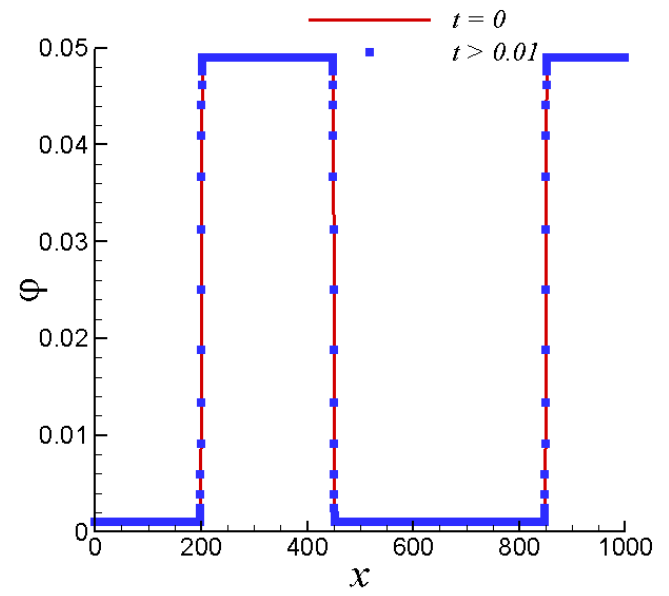

Fig. 25 (a) Multiple transition layers with levels of transition layers close to the energy wells $\varphi=0$ and $\varphi=0.05$ 


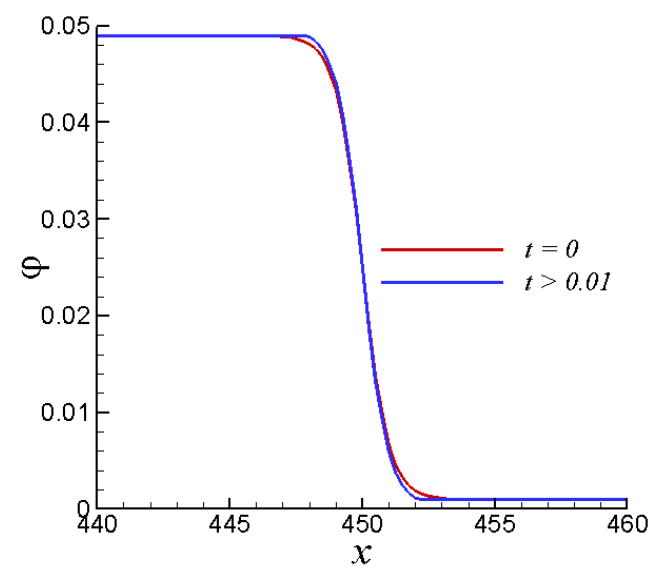

Fig. 25 (b) A section of the $\varphi$ profile showing a single transition layer

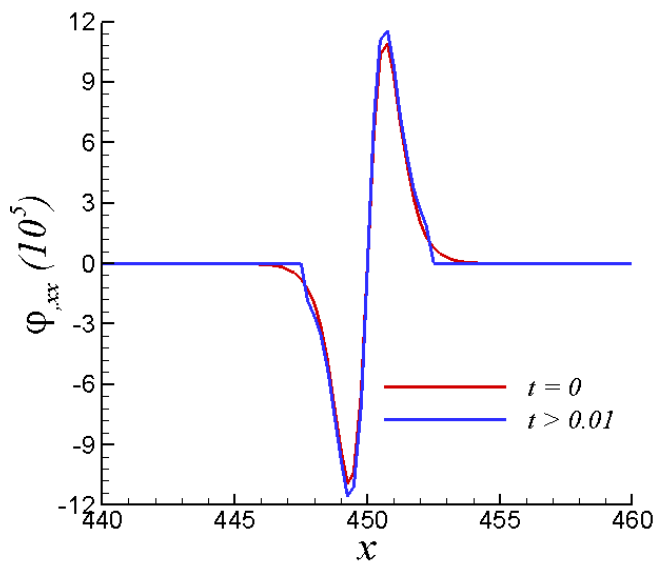

Fig. 25 (c) $\varphi_{x x}$ profile across a transition layer 


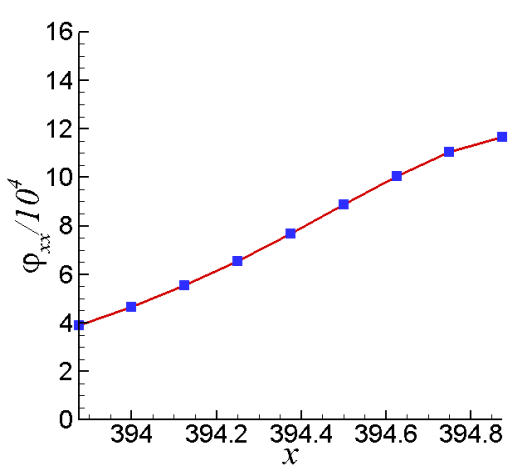

Fig. 26 (a)

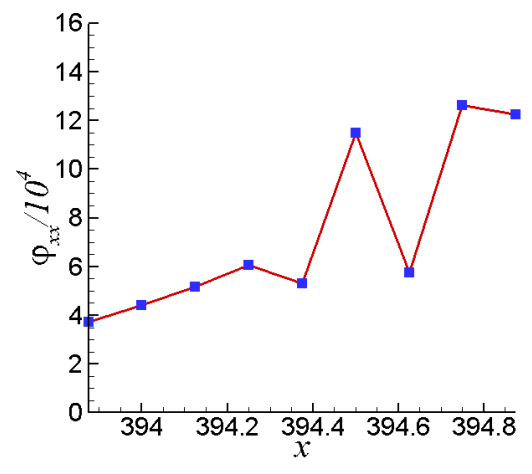

Fig. 26 (c)

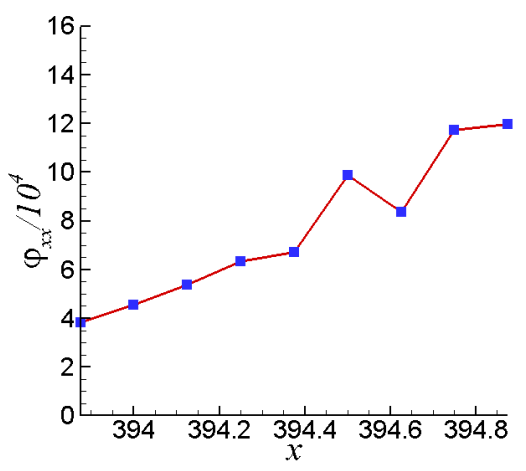

Fig. 26 (b)

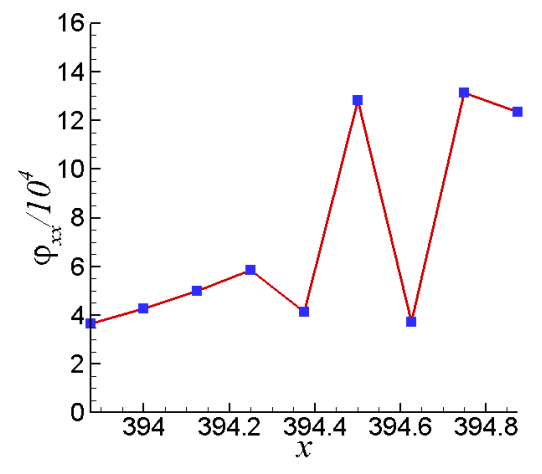

Fig. 26 (d)

Fig. 26 Evolution of $\varphi_{x x}$ in the absence of diffusion term in the governing equation (small section of the domain) 


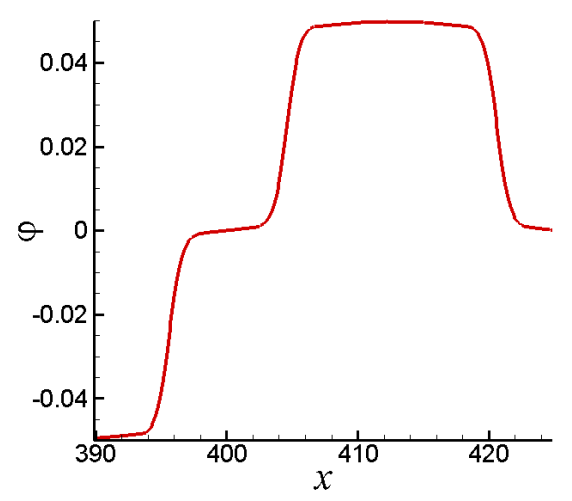

Fig. 27 (a)

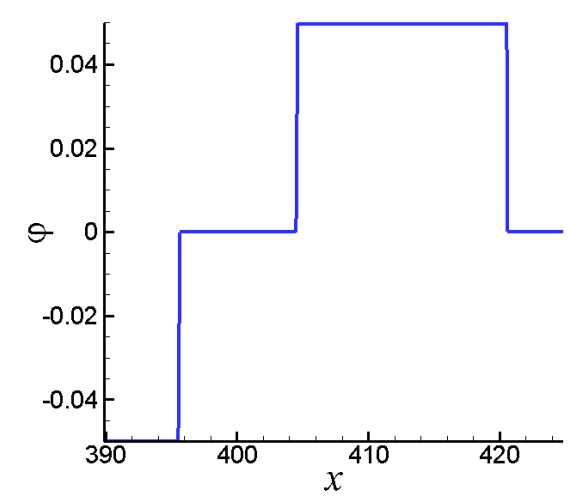

Fig. 27 (b)

Fig. 27 Transformation of the $\varphi$ profile in the absence of diffusion term in the governing equation (large section of the domain) 


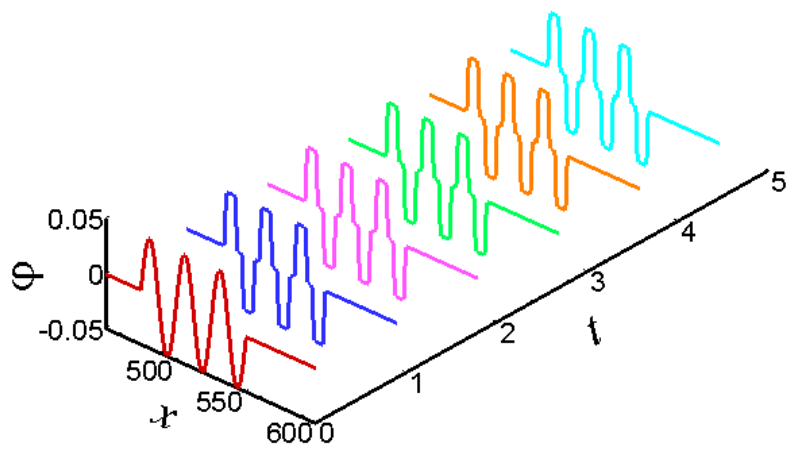

Fig. 28 Evolution of $\varphi$ for multiple sine waves case with Friedrichs

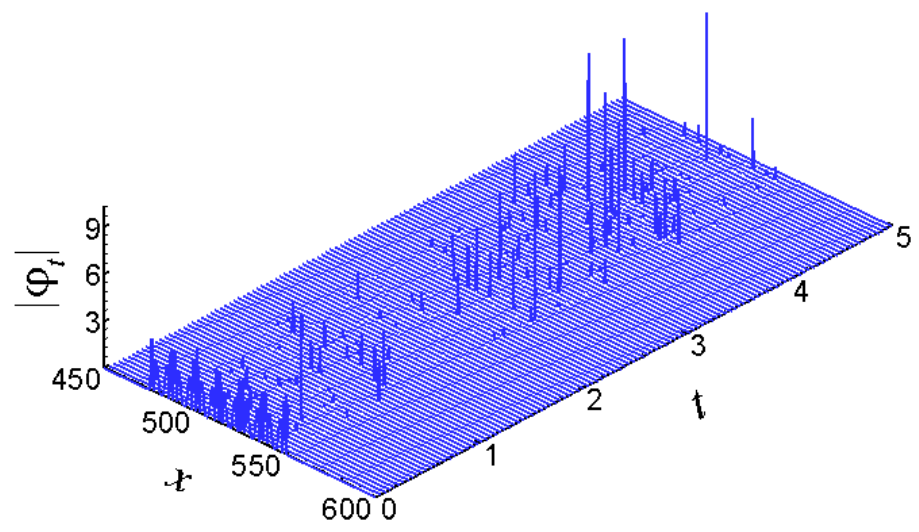

Fig. 29 Evolution of $\varphi_{t}$ for multiple sine waves case with Friedrichs 


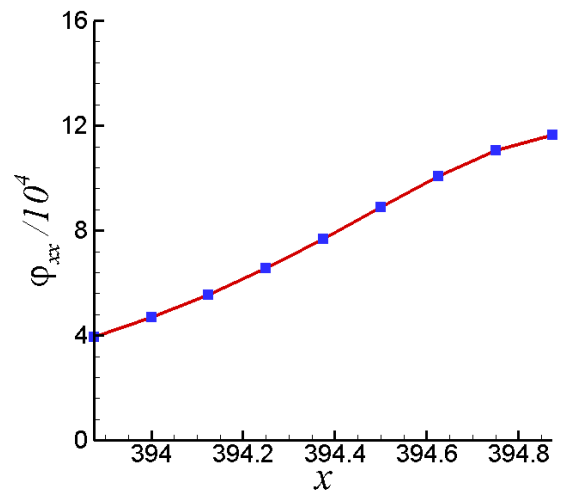

Fig. 30 (a)

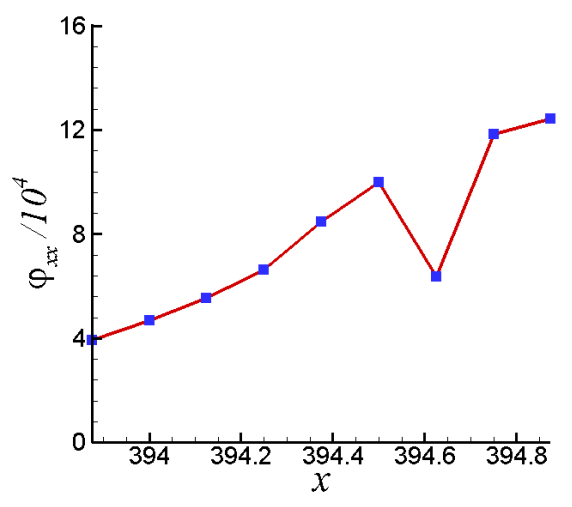

Fig. 30 (c)

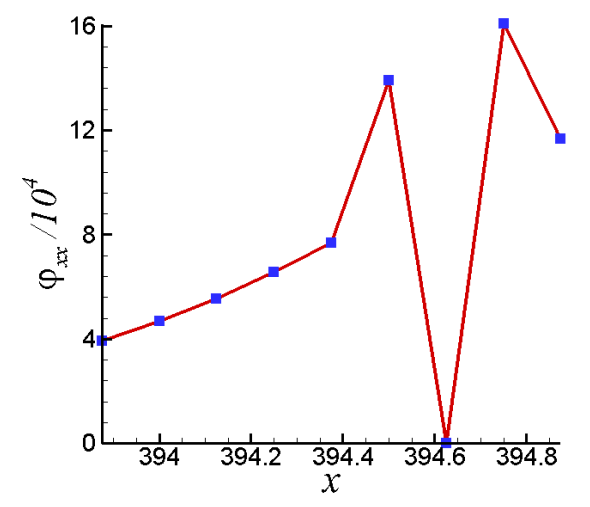

Fig. 30 (b)

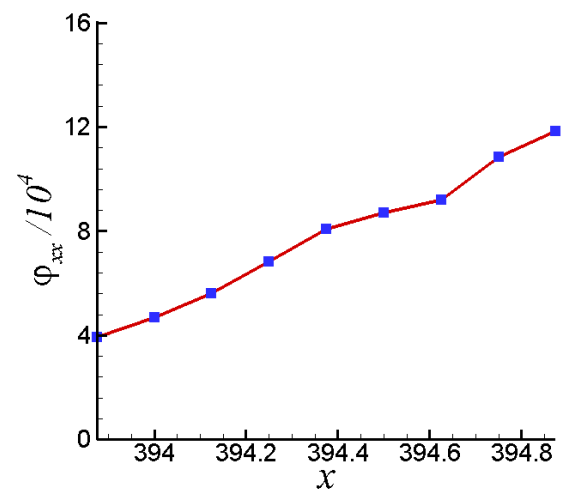

Fig. $30(d)$

Fig. 30 Evolution of $\varphi_{x x}$ with Friedrichs scheme (small section of the domain) 


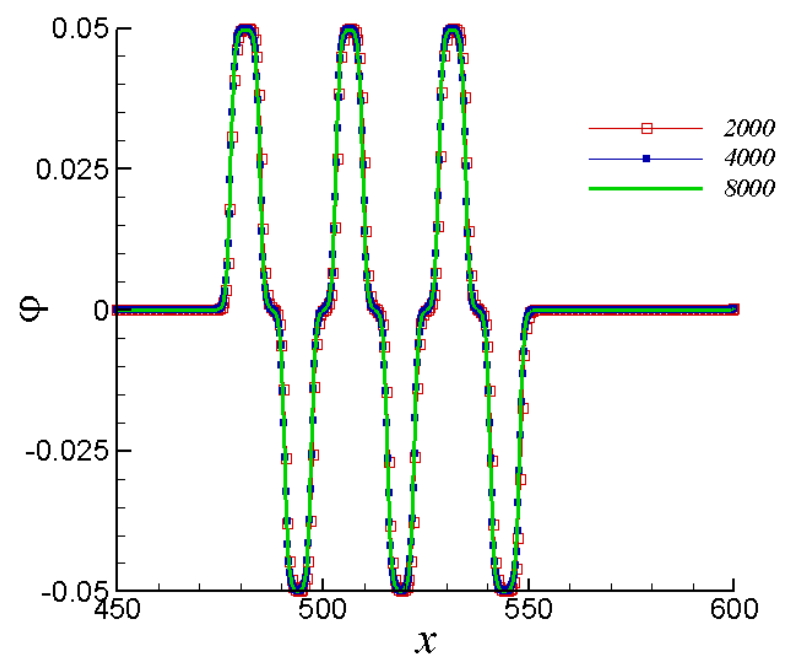

(a) plot of $\varphi$

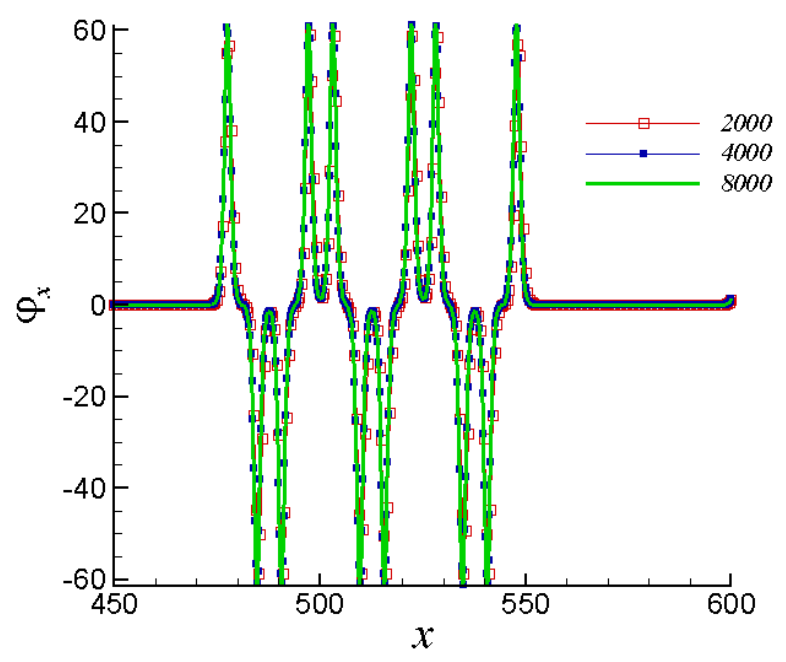

(b) plot of $\varphi_{x}$

Fig. 31 Effect of mesh refinement 

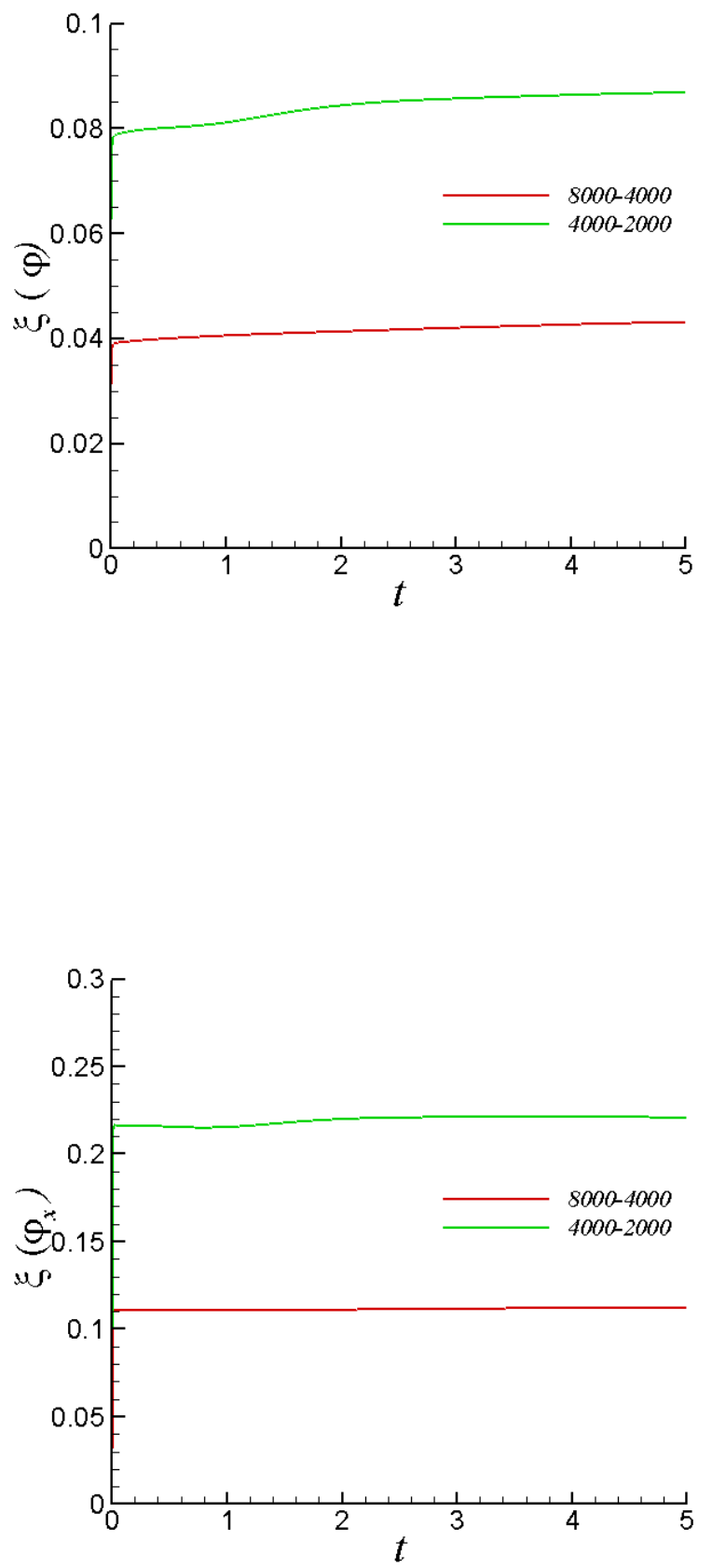

Fig. 32 Plot of variation of error norms with time 